\title{
HOMOGENIZATION OF UNBOUNDED FUNCTIONALS AND NONLINEAR ELASTOMERS. THE CASE OF THE FIXED CONSTRAINTS SET
}

\author{
Ludiano Carbone $^{1}$, Doina Cioranescu ${ }^{2}$, Riccardo De Arcangelis $^{1}$ And \\ Antonio Gaudiello ${ }^{3}$
}

\begin{abstract}
The paper is a continuation of a previous work of the same authors dealing with homogenization processes for some energies of integral type arising in the modeling of rubber-like elastomers. The previous paper took into account the general case of the homogenization of energies in presence of pointwise oscillating constraints on the admissible deformations. In the present paper homogenization processes are treated in the particular case of fixed constraints set, in which minimal coerciveness hypotheses can be assumed, and in which the results can be obtained in the general framework of $B V$ spaces. The classical homogenization result is established for Dirichlet with affine boundary data, Neumann, and mixed problems, by proving that the limit energy is again of integral type, gradient constrained, and with an explicitly computed homogeneous density.
\end{abstract}

Mathematics Subject Classification. 49J45, 49N20, 74Q05.

Received June 26, 2001. Revised May 28 and December 4, 2002.

\section{INTRODUCTION}

In this paper we continue the study, started in [7], of the homogenization of some classes of problems coming from the modeling of nonlinear elastomers. Our approach is based on the notion of unbounded functionals (cf. [9] for a presentation of the subject).

The mathematical models of the physical problem are recalled in Section 1.1 below. Their mathematical treatment is discussed in Section 1.2, where we also formulate the main results of this paper. We end this introductory section by a comparison of our results with some related ones.

\subsection{Sketch of the mathematical modeling of the physical problem}

Nonlinear elastomers are essentially materials which behave as rubber. The classical reference for the study of their behaviour is the book of L.R.G. Treloar published in several editions ( $c f$. [24]). Let us describe some examples of Treloar's modelings.

\footnotetext{
Keywords and phrases. Homogenization, gradient constrained variational problems, nonlinear elastomers

1 Università di Napoli "Federico II", Dipartimento di Matematica e Applicazioni "R. Caccioppoli", via Cintia, Complesso Monte S. Angelo, 80126 Napoli, Italy; e-mail: carbone@biol.dgbm.unina.it, dearcang@unina.it

${ }^{2}$ Université Pierre et Marie Curie (Paris VI), Laboratoire Jacques-Louis Lions, 4 Place Jussieu, 75252 Paris Cedex 05, France; e-mail: cioran@ann.jussieu.fr

3 Università di Cassino, Dipartimento di Automazione, Elettromagnetismo, Ingegneria dell'Informazione e Matematica Industriale, via G. Di Biasio 43, 03043 Cassino (FR), Italy; e-mail: gaudiell@unina.it
} 
Let $\lambda_{1}, \lambda_{2}, \lambda_{3}$ denote the three principal extension ratios along three mutually perpendicular axes, and let $W$ be the work of deformation or elastically stored free energy per unit of volume. It is clear that $\lambda_{1}>0, \lambda_{2}>0$, $\lambda_{3}>0$. In addition, they also satisfy the condition for constancy of volume $\lambda_{1} \lambda_{2} \lambda_{3}=1$. Then Treloar obtains the following expression of $W$ for homogeneous materials in some scalar cases:

a) for the simple extension $\left(\lambda_{1}=\lambda, \lambda_{2}=\lambda_{3}=\lambda^{-\frac{1}{2}}\right)$

$$
W=\frac{1}{2} C_{1}\left(\lambda^{2}+\frac{2}{\lambda}-3\right), \quad \lambda>0
$$

b) for the simple shear $\left(\lambda_{1}=\lambda, \lambda_{2}=1, \lambda_{3}=\frac{1}{\lambda}\right)$

$$
W=\frac{1}{2} C_{2}\left(\lambda-\frac{1}{\lambda}\right)^{2}, \quad \lambda>0
$$

c) for the simple extension with large deviations

$$
W=C_{3}\left(\lambda^{2}+\frac{2}{\lambda}-3\right)+C_{4}\left(\frac{1}{\lambda^{2}}+2 \lambda-3\right), \quad \lambda>0,
$$

where $C_{1}, \ldots, C_{4}$ are elastic constants. When the materials are nonhomogeneous, the constants $C_{1}, \ldots, C_{4}$ explicitly depend on the space variable $x$.

Consequently, the models lead to the introduction of bulk energies characterized by the presence of pointwise constraints on the gradients of the admissible deformations, and of singularities in the energy densities. For example, having in mind the previous models, one can consider functionals of the calculus of variations of the form (in one dimension, and in the scalar case)

$$
F(u)=\int_{a}^{b} f\left(x, u^{\prime}(x)\right) \mathrm{d} x
$$

where $u$ is a deformation variable of the material, and

or

$$
\begin{gathered}
f(x, z)= \begin{cases}\frac{1}{2} C_{1}(x)\left(z^{2}+\frac{2}{z}-3\right) & \text { if } z>0 \\
+\infty & \text { if } z \leq 0\end{cases} \\
f(x, z)= \begin{cases}\frac{1}{2} C_{2}(x)\left(z-\frac{1}{z}\right)^{2} & \text { if } z>0 \\
+\infty & \text { if } z \leq 0\end{cases}
\end{gathered}
$$

$$
f(x, z)= \begin{cases}C_{3}(x)\left(z^{2}+\frac{2}{z}-3\right)+C_{4}(x)\left(\frac{1}{z^{2}}+2 z-3\right) & \text { if } z>0 \\ +\infty & \text { if } z \leq 0\end{cases}
$$

Note that in this way the constraint conditions have been included in the energy densities.

Then, one has to study the minimization of these energy functionals under suitable boundary conditions.

An equation approach to this problem has been recently used in [2].

Rubber-like materials are widely used in industries. To render them more rigid, rubber composites filled with inactive (carbon black and/or silica) or periodically distributed active elements (piezoelectric, magnetic or conductive particles) are used, as well as mixtures of different types of rubber-like materials. In general, the size of the fillers is very small compared with the global size of the materials. So, it is natural enough to apply homogenization techniques to study these materials. Obviously constraint conditions on the gradients play a fundamental role in the development of the relevant homogenization processes. For standard methods in homogenization, we refer the reader to the classical book of Bensoussan et al. [3], and to the more recent ones $[4,15]$ and $[19]$ for further developments and general references on the subject. 


\subsection{Mathematical treatment of models and main results}

In this paper we propose a general mathematical framework for treating the models described in Section 1.1 in the case where the deformation variables depend on several space variables.

We analyze the behaviour of sequences of functionals by means of $\Gamma$-convergence theory. We obtain a description of the limit problem by a classical homogenization formula, and we deduce convergence results for the corresponding minimizing deformations.

Our approach is based on two steps. The first one consists in proving that, as the period of the mixture becomes smaller and smaller, the corresponding energy functional becomes more and more similar to an homogeneous one, i.e. invariant with respect to space translations. The second step consists in applying some representation results for homogeneous functionals.

The limit functional we obtain is unbounded, i.e. it is not necessarily finite on all the smooth possible deformations. In order to describe this limit, in the second step we apply some recent representation results for this kind of functionals (cf. $[10,12])$.

The results of the present paper have been announced in [8].

Let us present more precisely our results. Let $Y=] 0,1{ }^{n}$, and denote by $\mathcal{L}$ the $\sigma$-algebra of the Lebesgue measurable subsets of $\mathbf{R}^{n}$ and by $\mathcal{B}$ the $\sigma$-algebra of the Borel subsets of $\mathbf{R}^{n}$. Let us take an energy density $f$ satisfying

$$
\left\{\begin{array}{l}
f:(x, z) \in \mathbf{R}^{n} \times \mathbf{R}^{n} \mapsto f(x, z) \in[0,+\infty] \\
f \mathcal{L} \otimes \mathcal{B} \text { measurable, } \\
f(\cdot, z) Y \text {-periodic for every } z \in \mathbf{R}^{n}, f(x, \cdot) \text { convex for a.e. } x \in \mathbf{R}^{n} .
\end{array}\right.
$$

Assume furthermore that the sets describing the constraints are fixed in the sense that (for every $g: \mathbf{R}^{n} \rightarrow$ $[0,+\infty]$ we set $\left.\operatorname{dom} g=\left\{z \in \mathbf{R}^{n}: g(z)<+\infty\right\}\right)$

$$
\operatorname{dom} f(x, \cdot)=C \text { for a.e. } x \in \mathbf{R}^{n}
$$

for some convex set $C$, not necessarily bounded, and such that

$$
\operatorname{int}(C) \neq \emptyset \text {. }
$$

We suppose also that $f$ satisfies the following mild summability condition in the space variable

$$
f(\cdot, z) \in L^{1}(Y) \text { for every } z \in C
$$

as well as the linear coerciveness one

$$
c_{1}|z|-c_{2} \leq f(x, z) \text { for a.e. } x \in \mathbf{R}^{n} \text {, and every } z \in \mathbf{R}^{n}
$$

for some $c_{1}>0, c_{2} \in \mathbf{R}$.

In this setting we are able to carry out the homogenization processes for Neumann, Dirichlet, and mixed problems. We will present below some examples of the results we have obtained for these problems. It is worth while to point out that these results depend on a regularity index $q$ related to the admissible functions in the infimum problems. It is well known that the corresponding infima may actually depend on this $q$ (this feature is known as Lavrentieff phenomenon), and that such dependence may even survive the homogenization process (cf. $[16,18,20])$. So, for every $q \in[1,+\infty]$, we define $f_{\text {hom }}^{q}$ as the nonnegative, real extended energy density given by

$$
f_{\text {hom }}^{q}: z \in \mathbf{R}^{n} \mapsto \inf \left\{\int_{Y} f(y, z+\nabla v) \mathrm{d} y: v \in W_{\text {per }}^{1, q}(Y)\right\},
$$

where $W_{\text {per }}^{1, q}(Y)$ is the set of the $Y$-periodic functions in $W_{\text {loc }}^{1, q}\left(\mathbf{R}^{n}\right)$. Observe that $f_{\text {hom }}^{q}$ is convex and satisfies

$$
c_{1}|z|-c_{2} \leq f_{\text {hom }}^{q}(z) \text { for every } z \in \mathbf{R}^{n} .
$$

Classically, (0.5) gives the so called homogenization formula. 
Let us denote by sc $f_{\text {hom }}^{q}$ the lower semicontinuous envelope of $f_{\text {hom }}^{q}$ and by $\left(\mathrm{sc}^{-} f_{\text {hom }}^{q}\right)^{\infty}$ its recession function (cf. Sect. 2 for the definition of recession functions).

Then, in the case of Neumann minimum problems, we prove that for every $q \in[1,+\infty]$, every convex bounded open set $\left.\Omega \subseteq \mathbf{R}^{n}, \lambda \in\right] 0,+\infty[, r \in] 1, \frac{n}{n-1}\left[(r \in] 1,+\infty[\right.$ if $n=1)$, and $\beta \in L^{\infty}(\Omega)$ the values

$$
i_{h}^{N}(q, \Omega, \lambda, r, \beta)=\inf \left\{\int_{\Omega} f(h x, \nabla u) \mathrm{d} x+\lambda \int_{\Omega}|u|^{r} \mathrm{~d} x+\int_{\Omega} \beta u \mathrm{~d} x: u \in W^{1, q}(\Omega)\right\}(h \in \mathbf{N})
$$

converge to

$$
=\min \left\{\int_{\Omega} \mathrm{sc}^{-} f_{\text {hom }}^{q}(\nabla u) \mathrm{d} x+\int_{\Omega}\left(\mathrm{sc}^{-} f_{\text {hom }}^{q}\right)^{\infty}\left(\frac{\mathrm{d} D^{s} u}{\mathrm{~d}\left|D^{s} u\right|}\right) \mathrm{d}\left|D^{s} u\right|+\lambda \int_{\Omega}|u|^{r} \mathrm{~d} x+\int_{\Omega} \beta u \mathrm{~d} x: u \in B V(\Omega)\right\} .
$$

Moreover, if for every $h \in \mathbf{N}, \tilde{u}_{h} \in W^{1, q}(\Omega)$ satisfies

$$
\lim _{h \rightarrow+\infty}\left(\int_{\Omega} f\left(h x, \nabla \tilde{u}_{h}\right) \mathrm{d} x+\lambda \int_{\Omega}\left|\tilde{u}_{h}\right|^{r} \mathrm{~d} x+\int_{\Omega} \beta \tilde{u}_{h} \mathrm{~d} x-i_{h}^{N}(q, \Omega, \lambda, r, \beta)\right)=0,
$$

then $\left\{\tilde{u}_{h}\right\}$ is compact in $L^{1}(\Omega)$ and its converging subsequences converge in $L^{1}(\Omega)$ to solutions of $m_{\infty}^{N}(q, \Omega, \lambda, r, \beta)$ (cf. Th. 7.2). We refer to Section 2 for the notation used in (1.7).

Similar results hold under coerciveness assumptions of order $p \in] 1,+\infty]$, and in the framework of Sobolev spaces ( $c f$. Th. 7.3), in which also boundary terms can be taken into account.

Observe that, thanks to (1.2), the variational problems in (1.6) are actually formulated in spaces of functions satisfying the pointwise constraint $\nabla u(x) \in C$ for a.e. $x \in \Omega$. Since $f_{\text {hom }}^{q}$ may take the value $+\infty$, the problems in (1.7) also involve gradient constraints on the admissible functions.

In the case of Dirichlet minimum problems, we prove that for every $q \in[1,+\infty]$, every convex bounded open set $\Omega, \lambda \in] 0,+\infty[, r \in] 1, \frac{n}{n-1}\left[(r \in] 1,+\infty[\right.$ if $n=1), \beta \in L^{\infty}(\Omega), z_{0} \in \operatorname{int}(C), c \in \mathbf{R}$ the values $\left(u_{z_{0}}\right.$ is the linear function with gradient $z_{0} \in \mathbf{R}^{n}$ )

$$
i_{h}^{D}(q, \Omega, \lambda, r, \beta)=\inf \left\{\int_{\Omega} f(h x, \nabla u) \mathrm{d} x+\lambda \int_{\Omega}|u|^{r} \mathrm{~d} x+\int_{\Omega} \beta u \mathrm{~d} x: u \in u_{z_{0}}+c+W_{0}^{1, q}(\Omega)\right\}
$$

converge to

$$
\begin{aligned}
& m_{\infty}^{D}(q, \Omega, \lambda, r, \beta)=\min \left\{\int_{\Omega} \mathrm{sc}^{-} f_{\text {hom }}^{q}(\nabla u) \mathrm{d} x+\int_{\Omega}\left(\mathrm{sc}^{-} f_{\text {hom }}^{q}\right)^{\infty}\left(\frac{\mathrm{d} D^{s} u}{\mathrm{~d}\left|D^{s} u\right|}\right) \mathrm{d}\left|D^{s} u\right|\right. \\
& \left.+\int_{\partial \Omega}\left(\mathrm{sc}^{-} f_{\text {hom }}^{q}\right)^{\infty}\left(\left(u-u_{z_{0}}-c\right) \mathbf{n}_{\Omega}\right) \mathrm{d} \mathcal{H}^{n-1}+\lambda \int_{\Omega}|u|^{r} \mathrm{~d} x+\int_{\Omega} \beta u \mathrm{~d} x: u \in B V(\Omega)\right\}
\end{aligned}
$$

Moreover, if for every $h \in \mathbf{N}, \tilde{u}_{h} \in u_{z_{0}}+c+W_{0}^{1, q}(\Omega)$ satisfies

$$
\lim _{h \rightarrow+\infty}\left(\int_{\Omega} f\left(h x, \nabla \tilde{u}_{h}\right) \mathrm{d} x+\lambda \int_{\Omega}\left|\tilde{u}_{h}\right|^{r} \mathrm{~d} x+\int_{\Omega} \beta \tilde{u}_{h} \mathrm{~d} x-i_{h}^{D}(q, \Omega, \lambda, r, \beta)\right)=0,
$$

then $\left\{\tilde{u}_{h}\right\}$ is compact in $L^{1}(\Omega)$, and its converging subsequences converge in $L^{1}(\Omega)$ to solutions of $m_{\infty}^{D}(q, \Omega, \lambda, r, \beta)$ (cf. Th. 7.4). Let us notice that the above formula makes sense. Indeed, since every convex open set $\Omega$ has a locally Lipschitz boundary, the unit outward normal $\mathbf{n}_{\Omega}$ to $\partial \Omega$ exists $\mathcal{H}^{n-1}$-a.e. in $\partial \Omega\left(\mathcal{H}^{n-1}\right.$ is the $(n-1)$ dimensional Hausdorff measure).

Like for the Neumann problem, the above results hold also under coerciveness assumptions of order $p \in$ ] $1,+\infty]$, and in the framework of Sobolev spaces (cf. Th. 7.5). 
Both in the Neumann and Dirichlet cases, if a coerciveness condition of order $p \in] 1,+\infty]$ holds, if the regularity index $q$ agrees with $p$, and if furthermore

$$
f(x, \cdot) \text { is lower semicontinuous for a.e. } x \in \mathbf{R}^{n},
$$

then the limit density $f_{\text {hom }}^{p}$ is lower semicontinuous too. Therefore, $\mathrm{sc}^{-} f_{\text {hom }}^{p}=f_{\text {hom }}^{p}$, infima in (1.6) and (1.8) are attained, and consequently convergence results for minima hold.

In the case of mixed boundary conditions, we give convergence results in the framework of Sobolev spaces, under the following superlinear growth assumption

$$
\phi(z) \leq f(x, z) \leq a(x)+M \phi(z) \text { for a.e. } x \in \mathbf{R}^{n} \text {, and every } z \in \mathbf{R}^{n}
$$

for some $\phi: \mathbf{R}^{n} \rightarrow[0,+\infty]$ convex such that $\operatorname{int}(\operatorname{dom} \phi) \neq \emptyset$ and $\lim _{z \rightarrow \infty} \frac{\phi(z)}{|z|}=+\infty, a \in L_{\text {loc }}^{1}\left(\mathbf{R}^{n}\right) Y$-periodic, $M \geq 0$

We prove that for every convex open set $\Omega, \Gamma \subseteq \partial \Omega$ with $\mathcal{H}^{n-1}(\Gamma)>0, \beta \in L^{\infty}(\Omega), \gamma \in L^{\infty}(\partial \Omega)$, $z_{0} \in \operatorname{int}(\operatorname{dom} \phi), c \in \mathbf{R}$ the values

$$
i_{h}^{M}(\Omega, \Gamma, \beta, \gamma)=\inf \left\{\int_{\Omega} f(h x, \nabla u) \mathrm{d} x+\int_{\Omega} \beta u \mathrm{~d} x+\int_{\partial \Omega} \gamma u \mathrm{~d} \mathcal{H}^{n-1}: u \in W^{1,1}(\Omega), u=u_{z_{0}}+c \text { in } \Gamma\right\}
$$

converge to

$$
m_{\infty}^{M}(\Omega, \Gamma, \beta, \gamma)=\min \left\{\int_{\Omega} \operatorname{sc}^{-} f_{\text {hom }}^{1}(\nabla u) \mathrm{d} x+\int_{\Omega} \beta u \mathrm{~d} x+\int_{\partial \Omega} \gamma u \mathrm{~d} \mathcal{H}^{n-1}: u \in W^{1,1}(\Omega), u=u_{z_{0}}+c \text { in } \Gamma\right\},
$$

and that, if for every $h \in \mathbf{N}, \tilde{u}_{h} \in W^{1,1}(\Omega)$ satisfies $u=u_{z_{0}}+c$ in $\Gamma$ and

$$
\lim _{h \rightarrow+\infty}\left(\int_{\Omega} f\left(h x, \nabla \tilde{u}_{h}\right) \mathrm{d} x+\int_{\Omega} \beta \tilde{u}_{h} \mathrm{~d} x+\int_{\partial \Omega} \gamma \tilde{u}_{h} \mathrm{~d} \mathcal{H}^{n-1}-i_{h}^{M}(\Omega, \Gamma, \beta, \gamma)\right)=0,
$$

then $\left\{\tilde{u}_{h}\right\}$ is compact in $L^{1}(\Omega)$, and its converging subsequences converge in $L^{1}(\Omega)$ to solutions of $m_{\infty}^{M}(\Omega, \Gamma, \beta, \gamma)$ (cf. Th. 7.6).

\subsection{Comparison with related results and plan of the paper}

Some energy functionals subject to constraints on the gradient have already been treated in the framework of the modeling of some elastic-plastic torsion problems. The modeling is developed in [22]. The corresponding homogenization problem was proposed in [3] (Chap. 1, Sect. 17), where also a conjecture on its solution was formulated: it predicted the validity of the homogenization formula for the limit problem. For this model, the constraints are essentially spheres centred in the origin and with a periodic radius, and moreover the energy densities are bounded where they are finite. So, the model constraint to be homogenized is given by

$$
|\nabla u(x)| \leq \varphi(x) \text { for a.e. } x,
$$

where $\varphi$ is a periodic measurable function. The model energy density is given by

$$
f(x, z)= \begin{cases}a(x)|z|^{2} & \text { if }|z| \leq \varphi(x) \\ +\infty & \text { if }|z|>\varphi(x),\end{cases}
$$

where $a$ is a periodic measurable function bounded from above and below by positive constants.

Several situations have been analyzed in literature depending on different properties of the radius $\varphi$ (cf. [14,17], and the references quoted therein). The more recent results in this setting are contained in [11]. They recover the previous ones, if boundedness of the radii is assumed. 
In several results of [11] assumptions on the radii were left the out of consideration. Cases in which the radii are not bounded could also be taken into account, but in the functional setting of $W^{1, p}$ Sobolev spaces, with $p$ bigger than the space dimension.

By using the methodology described in Section 1.2, in [7] homogenization problems have been approached under very general assumptions on the constraints, and on the behaviour of the energy densities, to cover also the models proposed in Section 1.1. So, both of them have been allowed to quickly oscillate, and gradient constraints have been described just by convex sets not necessarily bounded. But in turn, high order coerciveness conditions had to be assumed, leading again to the functional setting of $W^{1, p}$ Sobolev spaces, with $p$ bigger than the space dimension $n$.

This assumption on $p$ does not allow to consider some other interesting cases. For example, if $n \geq 2$, energies with quadratic growth at infinity as those described in Section 1.1 cannot be considered in this setting, even in the simplest case when the constraints are not oscillating.

The study of the case when $p<n$ presents several difficulties that are essentially linked to the loss of continuity of the admissible deformations. The present paper is a first attempt in this direction. We consider here the simple situation when the constraints are fixed, namely independent on the space variable. We use a new technique, based on unique extension properties of functionals treated in [13], that allows us to establish homogenization results in the general setting of $B V$ spaces, where strongly discontinuities are allowed. In this new framework we can also reduce the assumption on $p$ to the natural one $p \geq 1$.

The paper is organized as follows.

In Section 2 we recall some preliminary notions and results needed in the paper. In Section 3 we collect some preparatory results. In Sections 4, 5, and 6 the main homogenization results are proved in terms of $\Gamma$-convergence, respectively for energies with Neumann, Dirichlet, and mixed boundary conditions. Finally, in Section 7 we prove the convergence of minimum energies and of minimizing deformation sequences.

\section{Notation AND RECALLS}

\section{1. $B \boldsymbol{V}$ spaces and convex analysis}

Let $\Omega \subseteq \mathbf{R}^{n}$ be open. By $B V(\Omega)$ we denote the set of the functions in $L^{1}(\Omega)$ having distributional partial derivatives that are Borel measures with bounded total variations in $\Omega$. We refer, for example, to [1] (Chap. 3), and [25] (Chap. 5) for a complete treatment of such spaces.

For every $u \in B V(\Omega)$, we denote the $\mathbf{R}^{n}$-valued measure gradient of $u$ by $D u$, and the total variation of $D u$ by $|D u|$. Moreover, according to Lebesgue Decomposition Theorem, we have that

$$
D u(E)=\int_{E} \nabla u \mathrm{~d} x+D^{s} u(E) \text { for every Borel set } E \subseteq \Omega,
$$

where $\nabla u$ is the density of the absolutely continuous part of $D u$, and $D^{s} u$ is the singular part of $D u$, both with respect to Lebesgue measure. We also denote by $\frac{\mathrm{d} D^{s} u}{\mathrm{~d} \mid D^{s} u}$ the Radon-Nikodym derivative of $D^{s} u$ with respect to its total variation $\left|D^{s} u\right|$.

The functional $u \in B V(\Omega) \mapsto\|u\|_{L^{1}(\Omega)}+|D u|(\Omega)$ is a norm that makes $B V(\Omega)$ a Banach space.

If in addition $\Omega$ has Lipschitz boundary, then it turns out that the functions in $B V(\Omega)$ have traces on $\partial \Omega$ in the sense that for every $u \in B V(\Omega)$ there exists an element in $L^{1}(\partial \Omega)$, still denoted by $u$, such that

$$
\int_{\Omega} u \operatorname{div} \varphi \mathrm{d} x=-\int_{\Omega} \varphi \cdot \mathrm{d} D u+\int_{\partial \Omega} \varphi \cdot \mathbf{n}_{\Omega} u \mathrm{~d} \mathcal{H}^{n-1} \text { for every } \varphi \in\left(C^{1}\left(\mathbf{R}^{n}\right)\right)^{n} .
$$

We also recall that, if $\Omega^{\prime}$ is another open set such that $\bar{\Omega} \subseteq \Omega^{\prime}$, and $v \in B V\left(\Omega^{\prime} \backslash \bar{\Omega}\right)$, then the function $w$, defined a.e. in $\Omega^{\prime}$ by setting $w=u$ in $\Omega$ and $w=v$ in $\Omega^{\prime} \backslash \bar{\Omega}$, is in $B V\left(\Omega^{\prime}\right)$. Moreover, by (2.1) it follows that

$$
D w(E)=\int_{E}(v-u) \mathbf{n}_{\Omega} \mathrm{d} \mathcal{H}^{n-1} \text { for every Borel set } E \subseteq \partial \Omega .
$$


Finally, we recall that when $\Omega$ has Lipschitz boundary, $B V(\Omega)$ compactly embeds in $L^{r}(\Omega)$ for every $r \in$ $\left[1, \frac{n}{n-1}[(r \in[1,+\infty[\right.$ if $n=1)$.

By $B V_{\text {loc }}\left(\mathbf{R}^{n}\right)$ we denote the set of the functions in $L_{\text {loc }}^{1}\left(\mathbf{R}^{n}\right)$ that are in $B V(\Omega)$ for every bounded open set $\Omega$. We recall that $B V_{\text {loc }}\left(\mathbf{R}^{n}\right)$ is a Fréchet space.

We now recall some basics of convex analysis. We refer for example to [23] (Part I and Part II) for a more complete exposition of the matter.

For every convex set $C \subseteq \mathbf{R}^{n}$ we denote by $\operatorname{ri}(C)$ the relative interior of $C$, i.e. the set of the interior points of $C$ in the topology of the smallest affine subset containing $C$. We recall that, for every convex set $C \subseteq \mathbf{R}^{n}$, $\operatorname{ri}(C) \neq \emptyset$, and that $\operatorname{ri}(C)=\operatorname{int}(C)$ provided $\operatorname{int}(C) \neq \emptyset$. Moreover, we also have that

$$
z_{0}+t\left(z-z_{0}\right) \in \operatorname{ri}(C) \text { whenever } z_{0} \in \operatorname{ri}(C) \text { and } z \in \bar{C} \text {. }
$$

Let $g: \mathbf{R}^{n} \rightarrow[0,+\infty]$ be convex. Then it is well known that dom $g$ is convex, that $g$ is lower semicontinuous in $\operatorname{ri}(\operatorname{dom} g)$, and that the restriction of $g$ to $\operatorname{ri}(\operatorname{dom} g)$ is continuous. In particular, if $\operatorname{int}(\operatorname{dom} g) \neq \emptyset$, then $g$ is continuous in $\operatorname{int}(\operatorname{dom} g)$.

If now $g: \mathbf{R}^{n} \rightarrow[0,+\infty]$, we denote by $g^{* *}$ the greatest convex lower semicontinuous function less than or equal to $g$, i.e.

$g^{* *}: z \in \mathbf{R}^{n} \mapsto \sup \left\{\phi(z): \phi: \mathbf{R}^{n} \rightarrow[0,+\infty]\right.$ convex and lower semicontinuous, $\phi(\xi) \leq g(\xi)$ for every $\left.\xi \in \mathbf{R}^{n}\right\}$.

It is clear that $g^{* *}$ is convex and lower semicontinuous, $g^{* *}(z) \leq g(z)$ for every $z \in \mathbf{R}^{n}$, and $g^{* *}=g$ provided $g$ itself is convex and lower semicontinuous.

In this paper, especially in connection with the definition of variational integrals on $B V$ spaces, we make use of recession functions. To define them properly, we recall that for a given $g: \mathbf{R}^{n} \rightarrow[0,+\infty]$ convex, and $z_{0} \in \operatorname{dom} g$, the $\operatorname{limit}_{\lim } \operatorname{lit}_{t \rightarrow \infty} \frac{g\left(z_{0}+t z\right)-g\left(z_{0}\right)}{t}$ exists for every $z \in \mathbf{R}^{n}$. Therefore we define the recession function of $g$ by

$$
g^{\infty}: z \in \mathbf{R}^{n} \mapsto \lim _{t \rightarrow+\infty} \frac{g\left(z_{0}+t z\right)-g\left(z_{0}\right)}{t} .
$$

It is well known that $g^{\infty}$ is positively 1-homogeneous, and that, if in addition $g$ is also lower semicontinuous, then the definition of $g^{\infty}$ does not depend on $z_{0}$ when it varies in dom $g$.

\subsection{Increasing set functions, unique extension, and lower semicontinuity results}

By $\mathcal{A}_{0}$ we denote the set of the bounded open subsets of $\mathbf{R}^{n}$.

For every couple $A, B$ of open subsets of $\mathbf{R}^{n}$ we write $A \subset \subset B$ if $\bar{A}$ is a compact subset of $B$.

Definition 2.1. Let $\alpha: \mathcal{A}_{0} \rightarrow[0,+\infty]$. We say that $\alpha$ is increasing if

$$
\alpha\left(A_{1}\right) \leq \alpha\left(A_{2}\right) \text { for every } A_{1}, A_{2} \in \mathcal{A}_{0} \text { such that } A_{1} \subseteq A_{2} .
$$

We denote by $\alpha_{-}$the inner regular envelope of $\alpha$ defined by

$$
\alpha_{-}: A \in \mathcal{A}_{0} \mapsto \sup \left\{\alpha(B): B \in \mathcal{A}_{0}, B \subset \subset A\right\},
$$

and say that $\alpha$ is inner regular if

$$
\alpha(A)=\alpha_{-}(A) \text { for every } A \in \mathcal{A}_{0} .
$$

It is clear that, if $\alpha: \mathcal{A}_{0} \rightarrow[0,+\infty]$, then $\alpha_{-}$is increasing and inner regular.

In the following, we consider functionals $\Phi$ defined in $\mathcal{A}_{0} \times U$ for some set $U$. In such case, given $(\Omega, u) \in$ $\mathcal{A}_{0} \times U$, we denote the inner regular envelope of $\Phi(\cdot, u)$ in $\Omega$ by $\Phi_{-}(\Omega, u)$, i.e. $\Phi_{-}(\Omega, u)=\Phi(\cdot, u)_{-}(\Omega)$. 
For every set $E \subseteq \mathbf{R}^{n}$, every function $u$ defined on $E, x_{0} \in \mathbf{R}^{n}$, and $\left.t \in\right] 0,+\infty\left[\right.$, by $T\left[x_{0}\right] u$ and $O_{t} u$ we denote the functions defined by

$$
T\left[x_{0}\right] u: x \in E-x_{0} \mapsto u\left(x+x_{0}\right), \quad O_{t} u: x \in \frac{1}{t} E \mapsto \frac{1}{t} u(t x) .
$$

The following inner regularity result is proved, also in a more general setting in [10] (Prop. 2.1).

Theorem 2.2. For every $\Omega \in \mathcal{A}_{0}$, let us consider a functional $\Phi(\Omega, \cdot): B V_{\mathrm{loc}}\left(\mathbf{R}^{n}\right) \rightarrow[0,+\infty]$ such that

$$
\text { for every } u \in B V_{\mathrm{loc}}\left(\mathbf{R}^{n}\right), \Phi(\cdot, u) \text { is increasing, }
$$

$$
\begin{gathered}
\liminf _{t \rightarrow 1^{-}} \Phi\left(\Omega, T\left[-x_{0}\right] O_{t} T\left[x_{0}\right] u\right) \geq \Phi(\Omega, u) \text { for every } \Omega \in \mathcal{A}_{0} \text { convex, } x_{0} \in \Omega, u \in B V_{\mathrm{loc}}\left(\mathbf{R}^{n}\right), \\
\limsup _{t \rightarrow 1^{+}} \Phi_{-}\left(x_{0}+t\left(\Omega-x_{0}\right), T\left[-x_{0}\right] O_{1 / t} T\left[x_{0}\right] u\right) \leq \Phi_{-}(\Omega, u) \\
\quad \text { for every } \Omega \in \mathcal{A}_{0} \text { convex, } x_{0} \in \Omega, u \in B V_{\mathrm{loc}}\left(\mathbf{R}^{n}\right) .
\end{gathered}
$$

Then

$$
\Phi(\Omega, u)=\Phi_{-}(\Omega, u) \text { for every } \Omega \in \mathcal{A}_{0} \text { convex, } u \in B V_{\mathrm{loc}}\left(\mathbf{R}^{n}\right)
$$

Let $U \subseteq L_{\text {loc }}^{1}\left(\mathbf{R}^{n}\right)$ be such that $T\left[x_{0}\right] u \in U$ whenever $u \in U$ and $x_{0} \in \mathbf{R}^{n}$.

Definition 2.3. Let $\Phi: \mathcal{A}_{0} \times U \rightarrow[0,+\infty]$. We say that $\Phi$ is

a) translation invariant if

$$
\Phi\left(\Omega-x_{0}, T\left[x_{0}\right] u\right)=\Phi(\Omega, u) \text { for every } \Omega \in \mathcal{A}_{0}, x_{0} \in \mathbf{R}^{n}, u \in U ;
$$

b) inner regular if for every $u \in U, \Phi(\cdot, u)$ is inner regular;

c) convex if for every $\Omega \in \mathcal{A}_{0}, \Phi(\Omega, \cdot)$ is convex;

d) $L_{\text {loc }}^{1}\left(\mathbf{R}^{n}\right)$-lower semicontinuous if for every $\Omega \in \mathcal{A}_{0}, \Phi(\Omega, \cdot)$ is $L_{\text {loc }}^{1}\left(\mathbf{R}^{n}\right)$-lower semicontinuous.

If $g: \mathbf{R}^{n} \rightarrow[0,+\infty]$ is convex and lower semicontinuous, we define the functional $\Phi_{g}$ as

$$
\Phi_{g}:(\Omega, u) \in \mathcal{A}_{0} \times B V_{\mathrm{loc}}\left(\mathbf{R}^{n}\right) \mapsto \int_{\Omega} g(\nabla u) \mathrm{d} x+\int_{\Omega} g^{\infty}\left(\frac{\mathrm{d} D^{s} u}{\mathrm{~d}\left|D^{s} u\right|}\right) \mathrm{d}\left|D^{s} u\right| .
$$

Then ( $c f$. for example [10]) it turns out that $\Phi_{g}$ is translation invariant, inner regular, convex, $L_{\text {loc }}^{1}(\Omega)$-lower semicontinuous, and

$$
\Phi_{g}(\Omega, u)=\int_{\Omega} g(\nabla u) \mathrm{d} x \text { for every }(\Omega, u) \in \mathcal{A}_{0} \times W_{\text {loc }}^{1,1}\left(\mathbf{R}^{n}\right) .
$$

Actually, $\Phi_{g}$ is the only functional on $\mathcal{A}_{0} \times B V_{\text {loc }}\left(\mathbf{R}^{n}\right)$ with these properties, as stated in the result below (cf. [12], Prop. 6.2).

Proposition 2.4. Let $g: \mathbf{R}^{n} \rightarrow[0,+\infty]$ be convex and lower semicontinuous, and let $\Phi_{g}$ be defined in (2.4). Then $\Phi_{g}$ is the only inner regular, translation invariant, convex, $L_{\text {loc }}^{1}\left(\mathbf{R}^{n}\right)$-lower semicontinuous functional from $\mathcal{A}_{0} \times B V_{\text {loc }}\left(\mathbf{R}^{n}\right)$ to $[0,+\infty]$ equal to $\int_{\Omega} g(\nabla u) \mathrm{d} x$ for every $(\Omega, u) \in \mathcal{A}_{0} \times C^{\infty}\left(\mathbf{R}^{n}\right)$.

Finally, we recall the following lower semicontinuity result ( $c f$. for example [5], Th. 4.1.1).

Theorem 2.5. Let $\Omega \in \mathcal{A}_{0}$ have Lipschitz boundary, and let $g: \Omega \times \mathbf{R}^{n} \rightarrow[0,+\infty]$ be $\mathcal{L} \otimes \mathcal{B}$ measurable. Assume that for a.e. $x \in \mathbf{R}^{n}, g(x, \cdot)$ is convex and lower semicontinuous. Then the functional

$$
u \in W^{1,1}(\Omega) \mapsto \int_{\Omega} g(x, \nabla u) \mathrm{d} x
$$

is sequentially weak- $W^{1,1}(\Omega)$-lower semicontinuous. 


\section{3. $\Gamma$-convergence, relaxation, and homogenization}

We recall now the definition of $\Gamma^{-}$-convergence (cf. [21], and [19], Chap. 4), and its fundamental feature concerning the convergence of sequences of minimum problems.

Let $(U, \tau)$ be a topological space satisfying the first countability axiom.

Definition 2.6. Let $\left\{E_{h}\right\}$ be a sequence of functionals from $U$ to $[-\infty,+\infty], u \in U$, and $\lambda^{\prime}, \lambda^{\prime \prime} \in[-\infty,+\infty]$. We say that $\lambda^{\prime}$ is the $\Gamma^{-}(\tau)$-lower limit of $\left\{E_{h}\right\}$ in $u$, and we write

$$
\lambda^{\prime}=\Gamma^{-}(\tau) \liminf _{h \rightarrow+\infty} E_{h}(u)
$$

if for every $\left\{v_{h}\right\} \subseteq U$ such that $v_{h} \stackrel{\tau}{\rightarrow} u$, one has

$$
\lambda^{\prime} \leq \liminf _{h \rightarrow+\infty} E_{h}\left(v_{h}\right)
$$

and if there exists $\left\{u_{h}\right\} \subseteq U$ such that $u_{h} \stackrel{\tau}{\rightarrow} u$ and

$$
\lambda^{\prime} \geq \liminf _{h \rightarrow+\infty} E_{h}\left(u_{h}\right)
$$

We say that $\lambda^{\prime \prime}$ is the $\Gamma^{-}(\tau)$-upper limit of $\left\{E_{h}\right\}$ in $u$, and we write

$$
\lambda^{\prime \prime}=\Gamma^{-}(\tau) \limsup _{h \rightarrow+\infty} E_{h}(u)
$$

if (2.6) and (2.7) hold with $\lambda^{\prime}$ and "liminf $\operatorname{in}_{h \rightarrow+\infty}$ " replaced by $\lambda^{\prime \prime}$ and "lim $\sup _{h \rightarrow+\infty}$ ", respectively. When $\lambda^{\prime}=\lambda^{\prime \prime}$ we say that $\left\{E_{h}\right\} \Gamma^{-}(\tau)$-converges in $u$, and we write

$$
\lambda^{\prime}=\lambda^{\prime \prime}=\Gamma^{-}(\tau) \lim _{h \rightarrow+\infty} E_{h}(u)
$$

We point out that, for every $u \in U$, the limits in (2.5) and (2.8) always exist, and we denote by $\Gamma^{-}(\tau) \liminf _{h \rightarrow+\infty} E_{h}, \quad \Gamma^{-}(\tau) \limsup _{h \rightarrow+\infty} E_{h}$ and, when existing, $\Gamma^{-}(\tau) \lim _{h \rightarrow+\infty} E_{h}$ the functionals $u \in U \mapsto \Gamma^{-}(\tau) \liminf _{h \rightarrow+\infty} E_{h}(u), u \in U \mapsto \Gamma^{-}(\tau) \lim \sup _{h \rightarrow+\infty} E_{h}(u)$, and $u \in U \mapsto \Gamma^{-}(\tau) \lim _{h \rightarrow+\infty} E_{h}(u)$, respectively.

We recall that

$$
\text { the functionals } \Gamma^{-}(\tau) \liminf _{h \rightarrow+\infty} E_{h} \text { and } \Gamma^{-}(\tau) \limsup _{h \rightarrow+\infty} E_{h} \text { are } \tau \text {-lower semicontinuous. }
$$

We say that the functionals $E_{h}$ are equicoercive if for every $t \in \mathbf{R}$ there exists a compact set $K_{t} \subseteq U$ such that $\cup_{h=1}^{+\infty}\left\{u \in U: E_{h}(u) \leq t\right\} \subseteq K_{t}$.

Theorem 2.7. Let $E_{1}, E_{2}, \ldots$ and $E$ be functionals from $U$ to $[-\infty,+\infty]$. Assume that, for every $u \in U$, the limit $\Gamma^{-}(\tau) \lim _{h \rightarrow+\infty} E_{h}(u)$ exists, that the functional $E$ is $\tau$-continuous, and that the functionals $E_{h}+E$ are equicoercive. Then $\Gamma^{-}(\tau) \lim _{h \rightarrow+\infty} E_{h}+E$ attains its minimum on $U$, and

$$
\min \left\{\Gamma^{-}(\tau) \lim _{h \rightarrow+\infty} E_{h}(v)+E(v): v \in U\right\}=\lim _{h \rightarrow+\infty} \inf \left\{E_{h}(v)+E(v): v \in U\right\} .
$$

Moreover, if $\left\{u_{h}\right\} \subseteq U$ is such that $\lim _{h \rightarrow+\infty}\left(E_{h}\left(u_{h}\right)+E\left(u_{h}\right)-\inf \left\{E_{h}(v)+E(v): v \in U\right\}\right)=0$, and $u_{h} \stackrel{\tau}{\rightarrow} u$, then $u$ is a solution of $\min \left\{\Gamma^{-}(\tau) \lim _{h \rightarrow+\infty} E_{h}(v)+E(v): v \in U\right\}$. 
As particular case, given $E: U \rightarrow[-\infty,+\infty]$, by setting $E_{h}=E$ for every $h \in \mathbf{N}$, it follows that the limit $\Gamma^{-}(\tau) \lim _{h \rightarrow+\infty} E_{h}$ exists on $U$, and that it agrees with the $\tau$-lower semicontinuous envelope of $E$, i.e. with the greatest $\tau$-lower semicontinuous functional less than or equal to $E$. We denote such functional by $\mathrm{sc}^{-}(\tau) E$.

When $(U, \tau)$ agrees with $\mathbf{R}^{n}$ endowed with its natural topology, we omit the indication of the topology in the $\mathrm{sc}^{-}$operator.

We recall that, if $g: \mathbf{R}^{n} \rightarrow[0,+\infty]$ is convex, then $\mathrm{sc}^{-} g$ too is convex, that ri $\left(\operatorname{dom}\left(\mathrm{sc}^{-} g\right)\right)=\operatorname{ri}(\operatorname{dom} g)$, and that for every $z_{0} \in \operatorname{dom} g$ the $\operatorname{limit}_{\lim _{t \rightarrow 1^{-}}} g\left((1-t) z_{0}+t z\right)$ exists and

$$
\mathrm{sc}^{-} g(z)=\lim _{t \rightarrow 1^{-}} g\left((1-t) z_{0}+t z\right) \text { for every } z \in \mathbf{R}^{n} .
$$

Let $g: \mathbf{R}^{n} \rightarrow[0,+\infty]$ be Borel, $u_{0} \in W_{\text {loc }}^{1,1}\left(\mathbf{R}^{n}\right)$, and, for every $\Omega \in \mathcal{A}_{0}$, let $\Psi_{g}\left(\Omega, u_{0}, \cdot\right)$ be defined by

$$
\Psi_{g}\left(\Omega, u_{0}, \cdot\right): u \in L_{\text {loc }}^{1}\left(\mathbf{R}^{n}\right) \mapsto \begin{cases}\int_{\Omega} g(\nabla u) \mathrm{d} x & \text { if } u \in u_{0}+W_{0}^{1, \infty}(\Omega) \\ +\infty & \text { otherwise. }\end{cases}
$$

We recall the following relaxation result (cf. [11], Th. 3.4).

Theorem 2.8. Let $g: \mathbf{R}^{n} \rightarrow[0,+\infty]$ be a Borel function such that $\operatorname{dom} g$ is convex and $\operatorname{int}(\operatorname{dom} g) \neq \emptyset$, and let $\Psi_{g}$ be defined by (2.11). Assume that

for every compact set $K \subseteq \operatorname{int}(\operatorname{dom} g)$ there exists $M_{K}>0$ such that $g(z) \leq M_{K}$ for every $z \in K$, and that

for every bounded set $L \subseteq \operatorname{dom} g$ there exists $z_{L} \in \operatorname{int}(\operatorname{dom} g)$ such that the function $t \in[0,1] \mapsto g\left((1-t) z_{L}+t z\right)$ is upper semicontinuous at $t=1$ uniformly for $z \in L$.

Then

$$
\begin{gathered}
\operatorname{sc}^{-}\left(L^{1}(\Omega)\right) \Psi_{g}\left(\Omega, u_{z_{0}}+c, u\right) \\
=\int_{\Omega} g^{* *}(\nabla u) \mathrm{d} x+\int_{\Omega}\left(g^{* *}\right)^{\infty}\left(\frac{\mathrm{d} D^{s} u}{\mathrm{~d}\left|D^{s} u\right|}\right) \mathrm{d}\left|D^{s} u\right|+\int_{\partial \Omega}\left(g^{* *}\right)^{\infty}\left(\left(u_{z_{0}}+c-u\right) \mathbf{n}_{\Omega}\right) \mathrm{d} \mathcal{H}^{n-1} \\
\text { for every } \Omega \in \mathcal{A}_{0} \text { convex, } z_{0} \in \operatorname{int}(\operatorname{dom} g), c \in \mathbf{R}, u \in B V(\Omega) .
\end{gathered}
$$

We now define the functionals that we study in the present paper.

Let $f$ be as in (1.1), and let $C \subseteq \mathbf{R}^{n}$ be convex and satisfying (1.2). For every $h \in \mathbf{N}, q \in[1,+\infty]$, and $\Omega \in \mathcal{A}_{0}$ we define the following functionals

$$
\begin{gathered}
F_{h}(\Omega, \cdot): u \in L_{\mathrm{loc}}^{1}\left(\mathbf{R}^{n}\right) \mapsto \begin{cases}\int_{\Omega} f(h x, \nabla u) \mathrm{d} x & \text { if } u \in W_{\mathrm{loc}}^{1, q}\left(\mathbf{R}^{n}\right) \\
+\infty & \text { otherwise, }\end{cases} \\
G_{h}(\Omega, \cdot): u \in L_{\mathrm{loc}}^{\infty}\left(\mathbf{R}^{n}\right) \mapsto F_{h}(\Omega, u),
\end{gathered}
$$

and their $\Gamma^{-}$-limits

$$
\begin{aligned}
& \left\{\begin{array}{l}
F^{\prime}(\Omega, \cdot): u \in L_{\mathrm{loc}}^{1}\left(\mathbf{R}^{n}\right) \mapsto \Gamma^{-}\left(L^{1}(\Omega)\right) \liminf _{h \rightarrow+\infty} F_{h}(\Omega, u), \\
F^{\prime \prime}(\Omega, \cdot): u \in L_{\mathrm{loc}}^{1}\left(\mathbf{R}^{n}\right) \mapsto \Gamma^{-}\left(L^{1}(\Omega)\right) \lim \sup _{h \rightarrow+\infty} F_{h}(\Omega, u),
\end{array}\right. \\
& \left\{\begin{array}{l}
G^{\prime}(\Omega, \cdot): u \in L_{\mathrm{loc}}^{\infty}\left(\mathbf{R}^{n}\right) \mapsto \Gamma^{-}\left(L^{\infty}(\Omega)\right) \liminf _{h \rightarrow+\infty} G_{h}(\Omega, u), \\
G^{\prime \prime}(\Omega, \cdot): u \in L_{\mathrm{loc}}^{\infty}\left(\mathbf{R}^{n}\right) \mapsto \Gamma^{-}\left(L^{\infty}(\Omega)\right) \lim \sup _{h \rightarrow+\infty} G_{h}(\Omega, u) .
\end{array}\right.
\end{aligned}
$$


We observe explicitly that because of (1.2), if $h \in \mathbf{N}, \Omega \in \mathcal{A}_{0}$, and $u \in L_{\text {loc }}^{1}\left(\mathbf{R}^{n}\right)$ satisfy $F_{h}(\Omega, u)<+\infty$, then $u \in W_{\mathrm{loc}}^{1, q}\left(\mathbf{R}^{n}\right)$ and $\nabla u(x) \in C$ for a.e. $x \in \Omega$.

Because of (1.1) it soon follows that

$$
\Gamma^{-}\left(L^{1}(\Omega)\right) \liminf _{h \rightarrow+\infty} F_{h}(\Omega, \cdot), \Gamma^{-}\left(L^{1}(\Omega)\right) \limsup _{h \rightarrow+\infty} F_{h}(\Omega, \cdot) \text { are convex for every } \Omega \in \mathcal{A}_{0}
$$

and

$$
\Gamma^{-}\left(L^{1}(\cdot)\right) \liminf _{h \rightarrow+\infty} F_{h}(\cdot, u), \Gamma^{-}\left(L^{1}(\cdot)\right) \limsup _{h \rightarrow+\infty} F_{h}(\cdot, u) \text { are increasing for every } u \in L_{\mathrm{loc}}^{1}\left(\mathbf{R}^{n}\right)
$$

Moreover, the following properties hold (cf. for example [19], Th. 24.1)

$$
\begin{gathered}
F_{-}^{\prime}\left(\Omega-x_{0}, T\left[x_{0}\right] u\right)=F_{-}^{\prime}(\Omega, u) \quad F_{-}^{\prime \prime}\left(\Omega-x_{0}, T\left[x_{0}\right] u\right)=F_{-}^{\prime \prime}(\Omega, u) \\
\text { for every } \Omega \in \mathcal{A}_{0}, x_{0} \in \mathbf{R}^{n}, u \in L_{\mathrm{loc}}^{1}\left(\mathbf{R}^{n}\right) .
\end{gathered}
$$

We also set

$$
\begin{gathered}
g_{\mathrm{hom}}^{q}: z \in \mathbf{R}^{n} \mapsto \inf \left\{\int_{Y} f(y, z+\nabla v) \mathrm{d} y: v \in W_{\mathrm{per}}^{1, q}(Y) \cap L^{\infty}(Y)\right\} \\
=\left\{z \in \mathbf{R}^{n}: \text { there exists } v \in W_{\mathrm{per}}^{1, q}(Y) \cap L^{\infty}(Y) \text { with } \int_{Y}\left\{f(y, z+\nabla v)+f\left(y, 2 z_{0}-z-\nabla v\right)\right\} \mathrm{d} y<+\infty\right\},
\end{gathered}
$$

and recall the following representation result (cf. [7], Prop. 6.1).

Proposition 2.9. Let $f$ be as in (1.1), $q \in[1,+\infty], G^{\prime}$ and $G^{\prime \prime}$ be defined in (2.14), $g_{\mathrm{hom}}^{q}$ in (2.18), and $C^{q}(0)$ in (2.19) with $z_{0}=0$. Assume that $\operatorname{int}\left(C^{q}(0)\right) \neq \emptyset$. Then $g_{\mathrm{hom}}^{q}$ is convex, and

$$
G_{-}^{\prime}(\Omega, u)=G_{-}^{\prime \prime}(\Omega, u)=\int_{\Omega} \operatorname{sc}^{-} g_{\mathrm{hom}}^{q}(\nabla u) \mathrm{d} x \text { for every } \Omega \in \mathcal{A}_{0}, u \in \cup_{s>n} W_{\mathrm{loc}}^{1, s}\left(\mathbf{R}^{n}\right) .
$$

For every $q \in[1,+\infty], \Omega \in \mathcal{A}_{0}$ with Lipschitz boundary, and $\Gamma \subseteq \partial \Omega$ we set

$$
W_{0, \Gamma}^{1, q}(\Omega)=\left\{u \in W^{1, q}(\Omega): u=0 \mathcal{H}^{n-1} \text {-a.e. in } \Gamma\right\} .
$$

It is clear that $W_{0, \partial \Omega}^{1, q}(\Omega)=W_{0}^{1, q}(\Omega)$ for every $q \in[1,+\infty]$. We also recall that, if $\Gamma$ is $\mathcal{H}^{n-1}$-measurable, then $W_{0, \Gamma}^{1, q}(\Omega)$ is weak- $W^{1, q}(\Omega)$-closed, and that, if $\mathcal{H}^{n-1}(\Gamma)>0$, then the following Poincaré inequality holds (cf. for example [25], Cor. 4.2.3)

$$
\|u\|_{L^{1}(\Omega)} \leq C_{\Omega, \Gamma}\|\nabla u\|_{L^{1}(\Omega)} \text { for every } u \in W_{0, \Gamma}^{1,1}(\Omega),
$$

where $C_{\Omega, \Gamma} \geq 0$ depends only on $\Omega$ and $\Gamma$.

Let $f$ be as in (1.1). For every $h \in \mathbf{N}, q \in[1,+\infty], \Omega \in \mathcal{A}_{0}$ with Lipschitz boundary, $\Gamma \subseteq \partial \Omega$, and $u_{0} \in W_{\text {loc }}^{1,1}\left(\mathbf{R}^{n}\right)$ we define the following functionals

$$
\begin{gathered}
F_{0, h}\left(\Omega, \Gamma, u_{0}, \cdot\right): u \in L_{\mathrm{loc}}^{1}\left(\mathbf{R}^{n}\right) \mapsto \begin{cases}\int_{\Omega} f(h x, \nabla u) \mathrm{d} x & \text { if } u \in u_{0}+W_{0, \Gamma}^{1, q}(\Omega) \\
+\infty & \text { otherwise },\end{cases} \\
G_{0, h}\left(\Omega, \Gamma, u_{0}, \cdot\right): u \in L_{\mathrm{loc}}^{\infty}\left(\mathbf{R}^{n}\right) \mapsto F_{0, h}\left(\Omega, \Gamma, u_{0}, u\right),
\end{gathered}
$$


and their $\Gamma^{-}$-limits

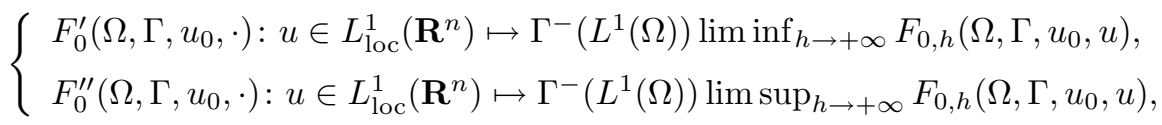

$$
\begin{aligned}
& \left\{\begin{array}{l}
G_{0}^{\prime}\left(\Omega, \Gamma, u_{0}, \cdot\right): u \in L_{\mathrm{loc}}^{\infty}\left(\mathbf{R}^{n}\right) \mapsto \Gamma^{-}\left(L^{\infty}(\Omega)\right) \liminf _{h \rightarrow+\infty} G_{0, h}\left(\Omega, \Gamma, u_{0}, u\right), \\
G_{0}^{\prime \prime}\left(\Omega, \Gamma, u_{0}, \cdot\right): u \in L_{\mathrm{loc}}^{\infty}\left(\mathbf{R}^{n}\right) \mapsto \Gamma^{-}\left(L^{\infty}(\Omega)\right) \lim _{\sup } \sup _{h \rightarrow+\infty} G_{0, h}\left(\Omega, \Gamma, u_{0}, u\right) .
\end{array}\right.
\end{aligned}
$$

The following representation result holds ( $c f .[7]$, Th. 6.2).

Theorem 2.10. Let $f$ be as in (1.1), $z_{0} \in \mathbf{R}^{n}, c \in \mathbf{R}, q \in[1,+\infty]$ and $\Omega \in \mathcal{A}_{0}$. Let $g_{\mathrm{hom}}^{q}$ be defined by (2.18), $C^{q}\left(z_{0}\right)$ in (2.19), and $G_{0}^{\prime}\left(\Omega, \partial \Omega, u_{z_{0}}+c, \cdot\right)$ and $G_{0}^{\prime \prime}\left(\Omega, \partial \Omega, u_{z_{0}}+c, \cdot\right)$ in (2.23). Let us assume that $\operatorname{int}\left(C^{q}\left(z_{0}\right)\right) \neq \emptyset$, then $g_{\mathrm{hom}}^{q}$ is convex, and

$$
\begin{gathered}
G_{0}^{\prime}\left(\Omega, \partial \Omega, u_{z_{0}}+c, u\right)=G_{0}^{\prime \prime}\left(\Omega, \partial \Omega, u_{z_{0}}+c, u\right)=\int_{\Omega} \mathrm{sc}^{-} g_{\mathrm{hom}}^{q}(\nabla u) \mathrm{d} x \\
\text { for every } u \in u_{z_{0}}+c+\cup_{s>n} W_{0}^{1, s}(\Omega) .
\end{gathered}
$$

\section{TECHNiCAL LEMMAS}

Let $f$ be as in (1.1). In the present section we prove some preparatory results, mainly of technical nature, that we will use in the sequel.

Proposition 3.1. Let $f$ be as in (1.1), $q \in[1,+\infty]$, and $f_{\mathrm{hom}}^{q}$ be defined in (1.5). Let $C \subseteq \mathbf{R}^{n}$ be such that (1.2) and (1.4) hold. Then $f_{\text {hom }}^{q}$ is convex, and

$$
C \subseteq \operatorname{dom} f_{\text {hom }}^{q} \subseteq \bar{C}
$$

Proof. The convexity of $f_{\text {hom }}^{q}$ is straightforward from (1.1).

By (1.4) it follows trivially that

$$
f_{\text {hom }}^{q}(z) \leq \int_{Y} f(y, z) \mathrm{d} y<+\infty \text { for every } z \in C,
$$

from which the left-hand side inequality follows.

Let now $z \in \operatorname{dom} f_{\text {hom }}^{q}$. Then there exists $v \in W_{\text {per }}^{1, q}(Y)$ such that $\int_{Y} f(y, z+\nabla v) \mathrm{d} y<+\infty$. Consequently, by (1.4), it follows that

$$
z+\nabla v(y) \in C \text { for a.e. } y \in Y \text {. }
$$

Since $\bar{C}$ is closed and convex, there exist two families $\left\{\alpha_{\theta}\right\}_{\theta \in \mathcal{T}} \subseteq \mathbf{R}^{n}$, and $\left\{\beta_{\theta}\right\}_{\theta \in \mathcal{T}} \subseteq \mathbf{R}$ such that $\zeta \in \bar{C}$ if and only if $\alpha_{\theta} \cdot \zeta+\beta_{\theta} \geq 0$ for every $\theta \in \mathcal{T}$. Therefore, by (3.1), we obtain that

$$
\alpha_{\theta} \cdot \int_{Y}(z+\nabla v) \mathrm{d} y+\beta_{\theta} \geq 0 \text { for every } \theta \in \mathcal{T} .
$$

By (3.2), the Gauss-Green Theorem, and the $Y$-periodicity of $v$ we deduce that

$$
\alpha_{\theta} \cdot z+\alpha_{\theta} \cdot \int_{\partial Y} v \mathbf{n}_{Y} \mathrm{~d} \mathcal{H}^{n-1}+\beta_{\theta}=\alpha_{\theta} \cdot z+\beta_{\theta} \geq 0 \text { for every } \theta \in \mathcal{T},
$$


from which we conclude that

This completes the proof.

$$
\operatorname{dom} f_{\text {hom }}^{q} \subseteq \bar{C}
$$

Lemma 3.2. Let $f$ be as in (1.1), $q \in[1,+\infty]$, and let $f_{\text {hom }}^{q}$ be defined in (1.5), and $g_{\text {hom }}^{q}$ in (2.18). Let $C \subseteq \mathbf{R}^{n}$ be convex such that (1.2), and (1.4) hold. Then

$$
\mathrm{sc}^{-} g_{\mathrm{hom}}^{q}(z)=\mathrm{sc}^{-} f_{\mathrm{hom}}^{q}(z) \text { for every } z \in \mathbf{R}^{n} .
$$

Proof. Since $f_{\text {hom }}^{q} \leq g_{\text {hom }}^{q}$, it is clear that

$$
\mathrm{sc}^{-} f_{\text {hom }}^{q}(z) \leq \mathrm{sc}^{-} g_{\text {hom }}^{q}(z) \text { for every } z \in \mathbf{R}^{n} .
$$

To prove the reverse inequality we first take $z \in \operatorname{ri}(C)$.

Let us observe that Proposition 3.1 yields $\operatorname{ri}\left(\operatorname{dom} f_{\text {hom }}^{q}\right)=\operatorname{ri}(C)$. Consequently $f_{\text {hom }}^{q}$, being convex, is lower semicontinuous in $\operatorname{ri}(C)$, and therefore $\operatorname{sc}^{-} f_{\text {hom }}^{q}(z)=f_{\text {hom }}^{q}(z)$. Because of this, we can assume that $f_{\text {hom }}^{q}(z)<$ $+\infty$, so that for every $\varepsilon>0$ there exists $u \in W_{\text {per }}^{1, q}(Y)$ such that

$$
f_{\mathrm{hom}}^{q}(z)+\varepsilon \geq \int_{Y} f(y, z+\nabla u) \mathrm{d} y
$$

For every $k \in \mathbf{N}$, set $u_{k}=\max \{\min \{u, k\},-k\}$. Then $u_{k} \in W_{\text {per }}^{1, q}(Y) \cap L^{\infty}(Y)$, and

$$
f_{\mathrm{hom}}^{q}(z)+\varepsilon \geq \int_{Y} f\left(y, z+\nabla u_{k}\right) \mathrm{d} y-\int_{\{y \in Y:|u(y)| \geq k\}} f(y, z) \mathrm{d} y \geq g_{\mathrm{hom}}^{q}(z)-\int_{\{y \in Y:|u(y)| \geq k\}} f(y, z) \mathrm{d} y,
$$

from which, letting first $k$ diverge and then $\varepsilon$ go to 0 , and by taking into account (1.4), we conclude that

$$
f_{\text {hom }}^{q}(z) \geq g_{\text {hom }}^{q}(z) \geq \operatorname{sc}^{-} g_{\text {hom }}^{q}(z) \text { for every } z \in \operatorname{ri}(C) \text {. }
$$

If now $z \in \bar{C}$, by the convexity of $f_{\text {hom }}^{q},(2.10),(2.3)$, and (3.4) we have that

$$
\begin{gathered}
\mathrm{sc}^{-} f_{\mathrm{hom}}^{q}(z)=\lim _{t \rightarrow 1^{-}} f_{\mathrm{hom}}^{q}\left(t z+(1-t) z_{0}\right) \geq \liminf _{t \rightarrow 1^{-}} \mathrm{sc}^{-} g_{\mathrm{hom}}^{q}\left(t z+(1-t) z_{0}\right) \geq \mathrm{sc}^{-} g_{\mathrm{hom}}^{q}(z) \\
\text { for every } z_{0} \in \operatorname{ri}(C), z \in \bar{C}
\end{gathered}
$$

In addition, since by Proposition 3.1 it follows that $f_{\text {hom }}^{q}(z)=+\infty$ for every $z \in \mathbf{R}^{n} \backslash \bar{C}$, we conclude that

$$
\mathrm{sc}^{-} f_{\text {hom }}^{q}(z)=+\infty \text { for every } z \in \mathbf{R}^{n} \backslash \bar{C} \text {. }
$$

By (3.3), (3.5), and (3.6), the lemma follows.

Proposition 3.3. Let $f$ be as in (1.1), $q \in[1,+\infty]$, $f_{\mathrm{hom}}^{q}$ be defined in (1.5), and let $\phi: \mathbf{R}^{n} \rightarrow[0,+\infty]$ be convex, and $a \in L_{\mathrm{loc}}^{1}\left(\mathbf{R}^{n}\right)$ be $Y$-periodic.

If

$$
\phi(z) \leq f(x, z) \text { for a.e. } x \in \mathbf{R}^{n} \text {, and every } z \in \mathbf{R}^{n} \text {, }
$$

then

$$
\mathrm{sc}^{-} \phi(z) \leq \mathrm{sc}^{-} f_{\text {hom }}^{q}(z) \text { for every } z \in \mathbf{R}^{n}
$$

If

$$
f(x, z) \leq a(x)+\phi(z) \text { for a.e. } x \in \mathbf{R}^{n} \text {, and every } z \in \mathbf{R}^{n} \text {, }
$$


then

$$
\mathrm{sc}^{-} f_{\mathrm{hom}}^{q}(z) \leq \int_{Y} a(y) \mathrm{d} y+\mathrm{sc}^{-} \phi(z) \text { for every } z \in \mathbf{R}^{n} .
$$

Proof. We first assume that (3.7) holds.

Let $z \in \mathbf{R}^{n}$, then (3.7) implies that

$$
\begin{gathered}
\inf \left\{\int_{Y} \operatorname{sc}^{-} \phi(z+\nabla v) \mathrm{d} y: v \in W_{\mathrm{per}}^{1, q}(Y)\right\} \leq \inf \left\{\int_{Y} \phi(z+\nabla v) \mathrm{d} y: v \in W_{\mathrm{per}}^{1, q}(Y)\right\} \\
\leq \inf \left\{\int_{Y} f(y, z+\nabla v) \mathrm{d} y: v \in W_{\mathrm{per}}^{1, q}(Y)\right\}=f_{\text {hom }}^{q}(z) .
\end{gathered}
$$

Now, $\mathrm{sc}^{-} \phi$ is convex and lower semicontinuous. Consequently the Gauss-Green Theorem and the Jensen's inequality provide that

$$
\operatorname{sc}^{-} \phi(z)=\operatorname{sc}^{-}\left(\int_{Y}(z+\nabla v) \mathrm{d} y\right) \leq \int_{Y} \operatorname{sc}^{-} \phi(z+\nabla v) \mathrm{d} y \text { for every } v \in W_{\text {per }}^{1,1}(Y),
$$

from which, together with (3.9), the first part of the proposition follows.

We now assume that (3.8) holds.

In this case, we first observe that (1.2) and (1.4) yield that

$$
f_{\mathrm{hom}}^{q}(z) \leq \int_{Y} f(y, z) \mathrm{d} y \text { for every } z \in \mathbf{R}^{n} .
$$

This, together with (3.8) entails that

$$
f_{\mathrm{hom}}^{q}(z) \leq \int_{Y} a(y) \mathrm{d} y+\phi(z) \text { for every } z \in \mathbf{R}^{n},
$$

from which also the second part of the proposition follows.

Proposition 3.4. Let $f$ be as in (1.1), $p \in[1,+\infty]$, and $f_{\mathrm{hom}}^{p}$ be defined in (1.5) with $q=p$. Assume that

$$
\begin{cases}\phi(z) \leq f(x, z) \text { for a.e. } x \in \mathbf{R}^{n}, \text { and every } z \in \mathbf{R}^{n} & \text { if } p=1 \\ c_{1}|z|^{p}-c_{2} \leq f(x, z) \text { for a.e. } x \in \mathbf{R}^{n}, \text { and every } z \in \mathbf{R}^{n} & \text { if } p \in] 1,+\infty[ \\ \operatorname{dom} f(x, \cdot) \subseteq\left\{z \in \mathbf{R}^{n}:|z| \leq R\right\} \text { for a.e. } x \in \mathbf{R}^{n} & \text { if } p=+\infty\end{cases}
$$

for some $\phi: \mathbf{R}^{n} \rightarrow[0,+\infty]$ convex such that $\lim _{z \rightarrow \infty} \phi(z) /|z|=+\infty, c_{1}>0, c_{2} \in \mathbf{R}, R>0$. Assume further that (1.9) holds. Then $f_{\mathrm{hom}}^{p}$ is lower semicontinuous, and

$$
f_{\text {hom }}^{p}(z)=\min \left\{\int_{Y} f(y, z+\nabla v) \mathrm{d} y: v \in W_{\mathrm{per}}^{1, p}(Y)\right\} \text { for every } z \in \mathbf{R}^{n} .
$$

Proof. Let $z \in \mathbf{R}^{n}$ and $\left\{z_{h}\right\} \subseteq \mathbf{R}^{n}$ be such that $z_{h} \rightarrow z$ and $\liminf _{h \rightarrow+\infty} f_{\text {hom }}^{p}\left(z_{h}\right)<+\infty$. Then there exists $\left\{h_{k}\right\} \subseteq \mathbf{N}$ strictly increasing such that for every $k \in \mathbf{N}$ there is $v_{k} \in W_{\text {per }}^{1, p}(Y)$ with $\lim _{k \rightarrow+\infty} \int_{Y} f\left(y, z_{h_{k}}+\right.$ $\left.\nabla v_{k}\right) \mathrm{d} y=\liminf _{h \rightarrow+\infty} f_{\text {hom }}^{p}\left(z_{h}\right)<+\infty$. Moreover, it is not restrictive to assume that $\int_{Y} v_{k} \mathrm{~d} y=0$ for every $k \in \mathbf{N}$.

This, together with the above coerciveness assumptions, and the Poincaré-Wirtinger inequality, implies that there exists $v \in W_{\text {per }}^{1, p}(Y)$ such that, up to subsequences, $v_{k} \rightarrow v$ in weak- $W^{1, p}(Y)$ (in weak ${ }^{*} W^{1, \infty}(Y)$ if $p=+\infty)$. 
Then, by (1.1), (1.9), and Theorem 2.5 we obtain that

$$
f_{\text {hom }}^{p}(z) \leq \int_{Y} f(y, z+\nabla v) \mathrm{d} x \leq \lim _{k \rightarrow+\infty} \int_{Y} f\left(y, z_{h_{k}}+\nabla v_{k}\right) \mathrm{d} y=\liminf _{h \rightarrow+\infty} f_{\text {hom }}^{p}\left(z_{h}\right),
$$

from which the lower semicontinuity of $f_{\text {hom }}^{p}$ follows.

In conclusion, by making use of the above coerciveness arguments and of Theorem 2.5, the classical direct methods of the calculus of variations ensure that for every $z \in \mathbf{R}^{n}$, the infimum in the definition of $f_{\text {hom }}^{p}$ is attained.

Lemma 3.5. Let $f$ be as in (1.1), and $q \in[1,+\infty]$. Let us assume that

i) $C \subseteq \mathbf{R}^{n}$ is convex satisfies (1.2) and (1.4), $0 \in \operatorname{ri}(C)$, and $\Omega \in \mathcal{A}_{0}, u \in W_{\text {loc }}^{1, \infty}\left(\mathbf{R}^{n}\right)$ are such that $\nabla u(x) \in \bar{C}$ for a.e. $x \in \Omega$;

or that

ii) $f$ satisfies (1.10) for some $\phi: \mathbf{R}^{n} \rightarrow\left[0,+\infty\left[\right.\right.$ convex with $0 \in \operatorname{ri}(\operatorname{dom} \phi), a \in L_{\text {loc }}^{1}\left(\mathbf{R}^{n}\right) Y$-periodic, $M \geq 0$, and $\Omega \in \mathcal{A}_{0}, u \in W_{\mathrm{loc}}^{1,1}\left(\mathbf{R}^{n}\right)$ are such that $\int_{\Omega} \mathrm{sc}^{-} \phi(\nabla u) \mathrm{d} x<+\infty$.

Then, for every $t \in\left[0,1\left[\right.\right.$, the integrals $\left\{\int f(h x, t \nabla u) \mathrm{d} x\right\}$ are equiabsolutely continuous in $\Omega$.

Proof. Let us first prove the thesis under the assumptions in i).

Let $t \in\left[0,1\left[\right.\right.$, and observe that, since $0 \in \operatorname{ri}(C)$ and $\nabla u \in\left(L^{\infty}(\Omega)\right)^{n}$, the convexity of $C$ provides the existence of $z_{1}, \ldots, z_{m} \in C$ such that $t \nabla u(x)$ belongs to the convex hull of $z_{1}, \ldots, z_{m}$ for a.e. $x \in \Omega$. Consequently, by the convexity of $f$, we deduce that

$$
f(h x, t \nabla u(x)) \leq \sum_{j=1}^{m} f\left(h x, \bar{z}_{j}\right) \text { for a.e. } x \in \Omega, \text { and every } h \in \mathbf{N}
$$

from which, together with (1.4) and the weak convergence in $L^{1}(\Omega)$ of $\left\{f\left(h \cdot, \bar{z}_{j}\right)\right\}$ for every $j \in\{1, \ldots, m\}$, the lemma under assumptions in i) follows.

Let us now assume that ii) holds.

Let $t \in\left[0,1\right.$. Then, since $\operatorname{dom} \phi$ is convex, $0 \in \operatorname{ri}(\operatorname{dom} \phi)$, and $\operatorname{ri}(\operatorname{dom} \phi)=\operatorname{ri}\left(\operatorname{dom}\left(\operatorname{sc}^{-} \phi\right)\right)$, we get $t \nabla u(x) \in$ ri $\left(\operatorname{dom}\left(\mathrm{sc}^{-} \phi\right)\right)$ for a.e. $x \in \Omega$. Consequently, by the convexity of $\phi$, we deduce that $\mathrm{sc}^{-} \phi(t \nabla u(x))=\phi(t \nabla u(x))$ for a.e. $x \in \Omega$. By virtue of this, the right-hand side of (1.10), and again the convexity of $\phi$ provide that

$$
\begin{gathered}
f(h x, t \nabla u(x)) \leq a(h x)+M \phi(t \nabla u(x))=a(h x)+M \mathrm{sc}^{-} \phi(t \nabla u(x)) \\
\leq a(h x)+M t \mathrm{sc}^{-} \phi(\nabla u(x))+M(1-t) \phi(0) \text { for a.e. } x \in \Omega, \text { and every } h \in \mathbf{N}
\end{gathered}
$$

from which, together with the finiteness of $\int_{\Omega} \mathrm{sc}^{-} \phi(\nabla u) \mathrm{d} x$, the weak convergence in $L^{1}(\Omega)$ of $\{a(h \cdot)\}$, and the finiteness of $\phi(0)$, the lemma follows also under assumptions in ii).

\section{The homogenization Result for the case of Neumann boundary conditions}

Let $f$ be as in (1.1), $F^{\prime}$ and $F^{\prime \prime}$ be defined in (2.13). In the present section we prove identity between $F^{\prime}$ and $F^{\prime \prime}$, together with an integral representation result for their common value.

Lemma 4.1. Let $f$ be as in (1.1), $q \in[1,+\infty]$, and $F^{\prime}$ be defined in (2.13). Let us assume that

i) $C \subseteq \mathbf{R}^{n}$ is convex satisfies (1.2) and (1.4), $0 \in \operatorname{ri}(C)$, and $\Omega \in \mathcal{A}_{0}, u \in W_{\text {loc }}^{1, \infty}\left(\mathbf{R}^{n}\right)$ are such that $F_{-}^{\prime}(\Omega, u)<+\infty$;

or that

ii) $f$ satisfies (1.10) for some $\phi: \mathbf{R}^{n} \rightarrow\left[0,+\infty\left[\right.\right.$ convex with $0 \in \operatorname{ri}(\operatorname{dom} \phi), a \in L_{\text {loc }}^{1}\left(\mathbf{R}^{n}\right) Y$-periodic, $M \geq 0$, and $\Omega \in \mathcal{A}_{0}, u \in W_{\mathrm{loc}}^{1,1}\left(\mathbf{R}^{n}\right)$ are such that $F_{-}^{\prime}(\Omega, u)<+\infty$. 
Then, for every $t \in\left[0,1\left[\right.\right.$, the integrals $\left\{\int f(h x, t \nabla u) \mathrm{d} x\right\}$ are equi-absolutely continuous in $\Omega$.

Proof. Let us first prove the thesis under the assumptions in i).

Since $F_{-}^{\prime}(\Omega, u)<+\infty$, fixed $A \in \mathcal{A}_{0}$ with $A \subset \subset \Omega$, by (1.2) there exists $\left\{u_{h}\right\} \subseteq L_{\text {loc }}^{1}\left(\mathbf{R}^{n}\right)$ such that $u_{h} \rightarrow u$ in $L^{1}(A)$, and $\liminf \inf _{h \rightarrow+\infty} F_{h}\left(A, u_{h}\right)<+\infty$. This, together with (1.2), provides $\left\{h_{k}\right\} \subseteq \mathbf{N}$ strictly increasing such that $\left\{u_{h_{k}}\right\} \subseteq W_{\text {loc }}^{1, q}\left(\mathbf{R}^{n}\right)$, and

$$
\text { for every } k \in \mathbf{N}, \nabla u_{h_{k}}(x) \in C \text { for a.e. } x \in A \text {. }
$$

Since $\bar{C}$ is closed and convex, there exist two families $\left\{\alpha_{\theta}\right\}_{\theta \in \mathcal{T}} \subseteq \mathbf{R}^{n}$, and $\left\{\beta_{\theta}\right\}_{\theta \in \mathcal{T}} \subseteq \mathbf{R}$ such that $\zeta \in \bar{C}$ if and only if $\alpha_{\theta} \cdot \zeta+\beta_{\theta} \geq 0$ for every $\theta \in \mathcal{T}$. Therefore, by (4.1), we obtain that

$$
\alpha_{\theta} \cdot \int_{A} \varphi \nabla u_{h_{k}} \mathrm{~d} x+\beta_{\theta} \geq 0 \text { for every } k \in \mathbf{N}, \theta \in \mathcal{T}, \varphi \in C_{0}^{1}(A) \text { with } \varphi \geq 0, \int_{A} \varphi \mathrm{d} x=1 .
$$

By (4.2), taking the limit as $k$ goes to $+\infty$, we deduce that

$$
\int_{A} \varphi \nabla u \mathrm{~d} x \in \bar{C} \text { for every } \varphi \in C_{0}^{1}(A) \text { with } \varphi \geq 0, \int_{A} \varphi \mathrm{d} x=1
$$

from which, letting $A$ increase to $\Omega$, we conclude that

$$
\nabla u(x) \in \bar{C} \text { for a.e. } x \in \Omega \text {. }
$$

Because of this, and of i) of Lemma 3.5, the lemma under assumptions in i) follows.

Let us now assume that ii) holds. Then, fixed $A \in \mathcal{A}_{0}$ with $A \subset \subset \Omega$, as before there exist $\left\{h_{k}\right\} \subseteq \mathbf{N}$ strictly increasing and $\left\{u_{k}\right\} \subseteq W_{\text {loc }}^{1, q}\left(\mathbf{R}^{n}\right)$ such that $u_{k} \rightarrow u$ in $L^{1}(A)$, and

$$
\liminf _{k \rightarrow+\infty} \int_{A} f\left(h_{k} x, \nabla u_{k}\right) \mathrm{d} x \leq F^{\prime}(A, u) \leq F_{-}^{\prime}(\Omega, u)<+\infty
$$

from which, making use of the left-hand side of (1.10) and of the $L^{1}(A)$-lower semicontinuity of $v \in W_{\text {loc }}^{1,1}\left(\mathbf{R}^{n}\right) \mapsto$ $\int_{A} \mathrm{sc}^{-} \phi(\nabla v) \mathrm{d} x$, it turns out that

$$
\begin{gathered}
\int_{A} \mathrm{sc}^{-} \phi(\nabla u) \mathrm{d} x \leq \liminf _{k \rightarrow+\infty} \int_{A} \mathrm{sc}^{-} \phi\left(\nabla u_{k}\right) \mathrm{d} x \leq \liminf _{k \rightarrow+\infty} \int_{A} \phi\left(\nabla u_{k}\right) \mathrm{d} x \\
\leq \liminf _{k \rightarrow+\infty} \int_{A} f\left(h_{k} x, \nabla u_{k}\right) \mathrm{d} x \leq F_{-}^{\prime}(\Omega, u)<+\infty \text { for every } A \in \mathcal{A}_{0} \text { with } A \subset \subset \Omega .
\end{gathered}
$$

Therefore,

$$
\int_{\Omega} \mathrm{sc}^{-} \phi(\nabla u) \mathrm{d} x<+\infty .
$$

Because of this, and of ii) of Lemma 3.5, the lemma under assumptions in ii) follows.

Lemma 4.2. Let $f$ be as in (1.1), $q \in[1,+\infty], F^{\prime}, F^{\prime \prime}$ be defined in (2.13), and $G^{\prime}, G^{\prime \prime}$ in (2.14). Then,

i) if $C \subseteq \mathbf{R}^{n}$ is convex and satisfies (1.2) and (1.4), one has

$$
G^{\prime}(\Omega, u)=F^{\prime}(\Omega, u) \text { for every } \Omega \in \mathcal{A}_{0}, u \in W_{\mathrm{loc}}^{1, \infty}\left(\mathbf{R}^{n}\right) ;
$$

ii) if $f$ satisfies (1.10) for some $\phi: \mathbf{R}^{n} \rightarrow[0,+\infty]$ convex, $a \in L_{\text {loc }}^{1}\left(\mathbf{R}^{n}\right) Y$-periodic, and $M \geq 0$, it results that

$$
G^{\prime \prime}(\Omega, u)=F^{\prime \prime}(\Omega, u) \text { for every } \Omega \in \mathcal{A}_{0}, u \in W_{\mathrm{loc}}^{1, q}\left(\mathbf{R}^{n}\right) \cap L^{\infty}(\Omega) .
$$


Proof. Let us prove the lemma under assumptions in i).

First of all, let us observe that it is not restrictive to assume that

$$
0 \in \operatorname{ri}(C)
$$

otherwise, taken $z_{0} \in \operatorname{ri}(C)$, we only have to consider the function $(x, z) \in \mathbf{R}^{n} \times \mathbf{R}^{n} \mapsto f\left(x, z_{0}+z\right)$ in place of $f$.

Let $\Omega \in \mathcal{A}_{0}, u \in W_{\text {loc }}^{1, \infty}\left(\mathbf{R}^{n}\right)$, and let us first prove that

$$
G^{\prime}(\Omega, u) \leq F^{\prime}(\Omega, u)
$$

To do this, we assume that $F^{\prime}(\Omega, u)<+\infty$.

Let us fix $t \in[0,1[$ and $\varepsilon>0$. By Lemma 4.1 there exists $\delta>0$ such that

$$
\int_{A} f(h x, t \nabla u) \mathrm{d} x<\varepsilon \text { for every } h \in \mathbf{N} \text {, whenever }|A|<\delta .
$$

By (4.3), (1.2), and (1.4) it follows that $F^{\prime}(\Omega, 0)<+\infty$. Then the finiteness of $F^{\prime}(\Omega, u)$ and of $F^{\prime}(\Omega, 0)$, and the convexity of $F^{\prime}(\Omega, \cdot)$ yield that $F^{\prime}(\Omega, t u)<+\infty$, too. Consequently, there exists $\left\{u_{t, h}\right\} \subseteq L_{\text {loc }}^{1}\left(\mathbf{R}^{n}\right)$ such that $u_{t, h} \rightarrow t u$ in $L^{1}(\Omega)$, and

$$
F^{\prime}(\Omega, t u)=\liminf _{h \rightarrow+\infty} F_{h}\left(\Omega, u_{t, h}\right) .
$$

Since $F^{\prime}(\Omega, t u)<+\infty$, it is easy to produce $\left\{h_{k}\right\} \subseteq \mathbf{N}$ strictly increasing such that $u_{t, h_{k}} \in W_{\text {loc }}^{1, q}\left(\mathbf{R}^{n}\right)$ for every $k \in \mathbf{N}$, and

$$
F^{\prime}(\Omega, t u)=\liminf _{k \rightarrow+\infty} \int_{\Omega} f\left(h_{k} x, \nabla u_{t, h_{k}}\right) \mathrm{d} x .
$$

Since clearly $u_{t, h_{k}} \rightarrow t u$ in measure in $\Omega$, for every $s \in \mathbf{N}$ there exists $\nu_{s} \in \mathbf{N}$ such that

$$
\left|\left\{x \in \Omega:\left|u_{t, h_{k}}(x)-t u(x)\right|>1 / s\right\}\right|<\delta \text { for every } k \geq \nu_{s},
$$

and it is not restrictive to assume that $\nu_{s+1}>\nu_{s}$. Because of this, if for every $k \in \mathbf{N}$ we call $s_{k}$ the only element in $\mathbf{N}$ such that $\nu_{s_{k}} \leq k<\nu_{s_{k}+1}$, we obtain that

$$
\left|\left\{x \in \Omega:\left|u_{t, h_{k}}(x)-t u(x)\right|>1 / s_{k}\right\}\right|<\delta \text { for every } k \geq \nu_{1} .
$$

For every $k \in \mathbf{N}$, we now take $\vartheta_{k} \in C^{1}(\mathbf{R})$ such that $0 \leq \vartheta_{k}^{\prime} \leq 1$ and

$$
\vartheta_{k}(r)= \begin{cases}-2 / s_{k} & \text { if } r<-3 / s_{k} \\ r & \text { if }-1 / s_{k} \leq r \leq 1 / s_{k} \\ 2 / s_{k} & \text { if } r>3 / s_{k}\end{cases}
$$

and define $\left\{w_{t, h}\right\}$ as

$$
w_{t, h}= \begin{cases}t u & \text { if } h \notin\left\{h_{k}\right\} \\ t u+\vartheta_{k}\left(u_{t, h_{k}}-t u\right) & \text { if } h=h_{k} \text { for some } k \in \mathbf{N}\end{cases}
$$

Then, it is clear that $w_{t, h} \in W_{\text {loc }}^{1, q}\left(\mathbf{R}^{n}\right) \cap L_{\mathrm{loc}}^{\infty}\left(\mathbf{R}^{n}\right)$ for every $h \in \mathbf{N}$, and that $w_{t, h} \rightarrow t u$ in $L^{\infty}(\Omega)$. Moreover, by the convexity of $f,(4.6),(4.7),(4.5)$, and the convexity of $F^{\prime}(\Omega, \cdot)$, we have that

$$
\begin{gathered}
G^{\prime}(\Omega, t u) \leq \liminf _{h \rightarrow+\infty} F_{h}\left(\Omega, w_{t, h}\right) \leq \liminf _{k \rightarrow+\infty} \int_{\Omega} f\left(h_{k} x, \nabla w_{t, h_{k}}\right) \mathrm{d} x \\
\leq \liminf _{k \rightarrow+\infty} \int_{\Omega} \vartheta_{k}^{\prime}\left(u_{t, h_{k}}-t u\right) f\left(h_{k} x, \nabla u_{t, h_{k}}\right) \mathrm{d} x+\limsup _{k \rightarrow+\infty} \int_{\left\{x \in \Omega:\left|u_{t, h_{k}}(x)-t u(x)\right|>1 / s_{k}\right\}} f\left(h_{k} x, t \nabla u\right) \mathrm{d} x
\end{gathered}
$$




$$
\leq F^{\prime}(\Omega, t u)+\varepsilon \leq t F^{\prime}(\Omega, u)+(1-t) F^{\prime}(\Omega, 0)+\varepsilon \text { for every } t \in[0,1[, \varepsilon>0 .
$$

In conclusion, once we observe that, since $u \in L^{\infty}(\Omega)$, then $t u \rightarrow u$ in $L^{\infty}(\Omega)$ as $t \rightarrow 1^{-}$, by (4.8) and (2.9), letting first $\varepsilon$ go to 0 and then $t$ increase to 1 , (4.4) follows.

Because of (4.4) the thesis follows, being obvious that $F^{\prime}(\Omega, u) \leq G^{\prime}(\Omega, u)$.

Finally, the proof of the lemma under assumptions in ii) comes by repeating word by word the above arguments. Actually, it is even simpler since, if $\Omega \in \mathcal{A}_{0}, u \in W_{\text {loc }}^{1, q}\left(\mathbf{R}^{n}\right) \cap L^{\infty}(\Omega)$ and $t \in[0,1[$ satisfy $F^{\prime \prime}(\Omega, t u)<+\infty$, then there exists $\left\{u_{t, h}\right\} \subseteq W_{\text {loc }}^{1, q}\left(\mathbf{R}^{n}\right)$ such that $u_{t, h} \rightarrow t u$ in $L^{1}(\Omega)$, and

$$
F^{\prime \prime}(\Omega, t u)=\limsup _{h \rightarrow+\infty} \int_{\Omega} f\left(h x, \nabla u_{t, h}\right) \mathrm{d} x .
$$

Because of this, there is no need to extract any subsequence $\left\{h_{k}\right\}$, and consequently for every $h \in \mathbf{N}, w_{t, h}$ is defined by $w_{t, h}=t u+\vartheta_{h}\left(u_{t, h}-t u\right)$.

Proposition 4.3. Let $f$ be as in (1.1), $q \in[1,+\infty], F^{\prime}$ and $F^{\prime \prime}$ be defined in (2.13), and $f_{\text {hom }}^{q}$ in (1.5). Let $C \subseteq \mathbf{R}^{n}$ be convex such that (1.2) $\div(1.4)$ hold. Then $f_{\text {hom }}^{q}$ is convex, and

$$
\begin{gathered}
F_{-}^{\prime}(\Omega, u)=F_{-}^{\prime \prime}(\Omega, u)=\int_{\Omega} \mathrm{sc}^{-} f_{\mathrm{hom}}^{q}(\nabla u) \mathrm{d} x+\int_{\Omega}\left(\mathrm{sc}^{-} f_{\mathrm{hom}}^{q}\right)^{\infty}\left(\frac{\mathrm{d} D^{s} u}{\mathrm{~d}\left|D^{s} u\right|}\right) \mathrm{d}\left|D^{s} u\right| \\
\text { for every } \Omega \in \mathcal{A}_{0}, u \in B V_{\mathrm{loc}}\left(\mathbf{R}^{n}\right) .
\end{gathered}
$$

Proof. As usual, it is not restrictive to assume that $0 \in \operatorname{int}(C)$.

Let $G^{\prime}$ and $G^{\prime \prime}$ be defined in (2.14), $g_{\text {hom }}^{q}$ in (2.18), and, for every $z_{0} \in \mathbf{R}^{n}, C^{q}\left(z_{0}\right)$ in (2.19). Then by i) of Lemma 4.2 we get that

$$
G^{\prime}(\Omega, u)=F^{\prime}(\Omega, u) \leq F^{\prime \prime}(\Omega, u) \leq G^{\prime \prime}(\Omega, u) \text { for every } \Omega \in \mathcal{A}_{0}, u \in W_{\text {loc }}^{1, \infty}\left(\mathbf{R}^{n}\right) .
$$

We now observe that it is easy to prove that $C \cap(-C) \subseteq C^{q}(0)$, from which, together with (1.3), we infer that $\operatorname{int}\left(C^{q}(0)\right) \neq \emptyset$, and hence, by Proposition 2.9, that $g_{\mathrm{hom}}^{q}$ is convex, and

$$
G_{-}^{\prime}(\Omega, u)=G_{-}^{\prime \prime}(\Omega, u)=\int_{\Omega} \operatorname{sc}^{-} g_{\text {hom }}^{q}(\nabla u) \mathrm{d} x \text { for every } \Omega \in \mathcal{A}_{0}, u \in W_{\text {loc }}^{1, \infty}\left(\mathbf{R}^{n}\right) .
$$

Then, by (4.9), (4.10), and Lemma 3.2 we deduce that

$$
F_{-}^{\prime}(\Omega, u)=F_{-}^{\prime \prime}(\Omega, u)=\int_{\Omega} \mathrm{sc}^{-} f_{\text {hom }}^{q}(\nabla u) \mathrm{d} x \text { for every } \Omega \in \mathcal{A}_{0}, u \in W_{\mathrm{loc}}^{1, \infty}\left(\mathbf{R}^{n}\right) .
$$

To conclude, due to (2.17), (2.15), (2.9) and (4.11), and by a double application of Proposition 2.4, first with $g=\mathrm{sc}^{-} f_{\text {hom }}^{q}$ and $\Phi$ equal to the restriction of $F_{-}^{\prime}$ to $\mathcal{A}_{0} \times C^{\infty}\left(\mathbf{R}^{n}\right)$, and then with $g=\mathrm{sc}^{-} f_{\text {hom }}^{q}$ and $\Phi$ equal to the restriction of $F_{-}^{\prime \prime}$ to $\mathcal{A}_{0} \times C^{\infty}\left(\mathbf{R}^{n}\right)$, the proposition follows.

Theorem 4.4. Let $f$ be as in (1.1), $q \in[1,+\infty], F^{\prime}$ and $F^{\prime \prime}$ be defined in (2.13), and $f_{\text {hom }}^{q}$ in (1.5). Let $C \subseteq \mathbf{R}^{n}$ be convex such that (1.2) $\div(1.4)$ hold. Then $f_{\text {hom }}^{q}$ is convex, and

$$
\begin{gathered}
F^{\prime}(\Omega, u)=F^{\prime \prime}(\Omega, u)=\int_{\Omega} \mathrm{sc}^{-} f_{\text {hom }}^{q}(\nabla u) \mathrm{d} x+\int_{\Omega}\left(\mathrm{sc}^{-} f_{\text {hom }}^{q}\right)^{\infty}\left(\frac{\mathrm{d} D^{s} u}{\mathrm{~d}\left|D^{s} u\right|}\right) \mathrm{d}\left|D^{s} u\right| \\
\text { for every } \Omega \in \mathcal{A}_{0} \text { convex, } u \in B V_{\mathrm{loc}}\left(\mathbf{R}^{n}\right) .
\end{gathered}
$$

Proof. By using (2.16), (2.9) and Proposition 4.3 it is easy to verify that $F^{\prime}$ and $F^{\prime \prime}$ fulfil the assumptions of Theorem 2.2. Therefore the thesis follows from Proposition 4.3, and Theorem 2.2. 


\section{The homogenization ReSUlt For the CASE OF DiRICHLET BOUNDARY CONDITIONS}

Let $f$ be as in (1.1), $\Omega \in \mathcal{A}_{0}, z_{0} \in \mathbf{R}^{n}, c \in \mathbf{R}$, and let $F_{0}^{\prime}\left(\Omega, \partial \Omega, u_{z_{0}}+c, \cdot\right)$ and $F_{0}^{\prime \prime}\left(\Omega, \partial \Omega, u_{z_{0}}+c, \cdot\right)$ be defined in $(2.22)$ with $\Gamma=\partial \Omega$. In this section we prove identity between $F_{0}^{\prime}\left(\Omega, \partial \Omega, u_{z_{0}}+c, \cdot\right)$ and $F_{0}^{\prime \prime}\left(\Omega, \partial \Omega, u_{z_{0}}+c, \cdot\right)$, and represent their common value.

Lemma 5.1. Let $f$ be as in (1.1), $q \in[1,+\infty], F_{0}^{\prime}$ and $F_{0}^{\prime \prime}$ be defined by (2.22), $f_{\mathrm{hom}}^{q}$ by (1.5), and $\Psi_{\mathrm{sc}^{-}} f_{\mathrm{hom}}^{q}$ by (2.11) with $g=\mathrm{sc}^{-} f_{\mathrm{hom}}^{q}$. Let $C \subseteq \mathbf{R}^{n}$ be convex such that (1.2) $\div(1.4)$ hold. Then $f_{\mathrm{hom}}^{q}$ is convex and

$$
\begin{gathered}
\int_{\Omega} \mathrm{sc}^{-} f_{\text {hom }}^{q}(\nabla u) \mathrm{d} x+\int_{\Omega}\left(\mathrm{sc}^{-} f_{\mathrm{hom}}^{q}\right)^{\infty}\left(\frac{\mathrm{d} D^{s} u}{\mathrm{~d}\left|D^{s} u\right|}\right) \mathrm{d}\left|D^{s} u\right|+\int_{\partial \Omega}\left(\operatorname{sc}^{-} f_{\mathrm{hom}}^{q}\right)^{\infty}\left(\left(u_{z_{0}}+c-u\right) \mathbf{n}_{\Omega}\right) \mathrm{d} \mathcal{H}^{n-1} \\
\leq F_{0}^{\prime}\left(\Omega, \partial \Omega, u_{z_{0}}+c, u\right) \leq F_{0}^{\prime \prime}\left(\Omega, \partial \Omega, u_{z_{0}}+c, u\right) \leq \operatorname{sc}^{-}\left(L^{1}(\Omega)\right) \Psi_{\mathrm{sc}^{-} f_{\text {hom }}^{q}}\left(\Omega, u_{z_{0}}+c, u\right) \\
\quad \text { for every } \Omega \in \mathcal{A}_{0} \text { with Lipschitz boundary, } z_{0} \in C, c \in \mathbf{R}, u \in B V_{\mathrm{loc}}\left(\mathbf{R}^{n}\right) .
\end{gathered}
$$

Proof. Let $g_{\text {hom }}^{q}$ and, for every $z_{0} \in \mathbf{R}^{n}, C^{q}\left(z_{0}\right)$ be defined in (2.18) and (2.19), respectively.

We observe now that, since $C \cap\left(2 z_{0}-C\right) \subseteq C^{q}\left(z_{0}\right)$, then $\operatorname{int}\left(C^{q}\left(z_{0}\right)\right) \neq \emptyset$ for every $z_{0} \in \operatorname{int}(C)$. Therefore, if $G_{0}^{\prime \prime}$ is defined by (2.23), from Theorem 2.10 and Lemma 3.2 we conclude that

$$
\begin{gathered}
F_{0}^{\prime \prime}\left(\Omega, \partial \Omega, u_{z_{0}}+c, u\right) \leq G_{0}^{\prime \prime}\left(\Omega, \partial \Omega, u_{z_{0}}+c, u\right)=\int_{\Omega} \mathrm{sc}^{-} g_{\mathrm{hom}}^{q}(\nabla u) \mathrm{d} x=\int_{\Omega} \mathrm{sc}^{-} f_{\mathrm{hom}}^{q}(\nabla u) \mathrm{d} x \\
\text { for every } \Omega \in \mathcal{A}_{0}, z_{0} \in \operatorname{int}(C), c \in \mathbf{R}, u \in u_{z_{0}}+c+W_{0}^{1, \infty}(\Omega) .
\end{gathered}
$$

Moreover, by using (0.4), it is easy to verify that

$$
F_{-}^{\prime}\left(\Omega^{\prime}, u\right)-\left|\Omega^{\prime} \backslash \Omega\right| \int_{Y} f\left(y, z_{0}\right) \mathrm{d} y \leq F_{0}^{\prime}\left(\Omega, \partial \Omega, u_{z_{0}}+c, u\right)
$$

for every $\Omega, \Omega^{\prime} \in \mathcal{A}_{0}$ with $\Omega \subset \subset \Omega^{\prime}, z_{0} \in C, c \in \mathbf{R}, u \in L_{\text {loc }}^{1}\left(\mathbf{R}^{n}\right)$ with $u=u_{z_{0}}+c$ a.e. in $\Omega^{\prime} \backslash \Omega$.

Let now $\Omega \in \mathcal{A}_{0}, z_{0} \in C, c \in \mathbf{R}, u \in B V_{\text {loc }}\left(\mathbf{R}^{n}\right)$. Let us change the values of $u$ in $\mathbf{R}^{n} \backslash \Omega$, and call again $u$ such extension, by setting $u=u_{z_{0}}+c$ in $\mathbf{R}^{n} \backslash \Omega$, then $u \in B V_{\text {loc }}\left(\mathbf{R}^{n}\right)$. Let $\left\{u_{h}\right\} \subseteq u_{z_{0}}+c+W_{0}^{1, \infty}(\Omega)$ be such that $u_{h} \rightarrow u$ in $L^{1}(\Omega)$, and

$$
\operatorname{sc}^{-}\left(L^{1}(\Omega)\right) \Psi_{\mathrm{sc}^{-} f_{\mathrm{hom}}^{q}}\left(\Omega, u_{z_{0}}+c, u\right) \geq \lim _{h \rightarrow+\infty} \int_{\Omega} \mathrm{sc}^{-} f_{\mathrm{hom}}^{q}\left(\nabla u_{h}\right) \mathrm{d} x .
$$

Then, by Proposition 2.3, (5.2), (2.9), (5.1), and (5.3), we have

$$
\begin{gathered}
\int_{\Omega^{\prime}} \operatorname{sc}^{-} f_{\text {hom }}^{q}(\nabla u) \mathrm{d} x+\int_{\Omega^{\prime}}\left(\operatorname{sc}^{-} f_{\mathrm{hom}}^{q}\right)^{\infty}\left(\frac{\mathrm{d} D^{s} u}{\mathrm{~d}\left|D^{s} u\right|}\right) \mathrm{d}\left|D^{s} u\right|-\left|\Omega^{\prime} \backslash \Omega\right| \int_{Y} f\left(y, z_{0}\right) \mathrm{d} y \\
\leq F_{0}^{\prime}\left(\Omega, \partial \Omega, u_{z_{0}}+c, u\right) \leq F_{0}^{\prime \prime}\left(\Omega, \partial \Omega, u_{z_{0}}+c, u\right) \\
\leq \liminf _{h \rightarrow+\infty} F_{0}^{\prime \prime}\left(\Omega, \partial \Omega, u_{z_{0}}+c, u_{h}\right) \leq \liminf _{h \rightarrow+\infty} \int_{\Omega} \operatorname{sc}^{-} f_{\text {hom }}^{q}\left(\nabla u_{h}\right) \mathrm{d} x \leq \operatorname{sc}^{-}\left(L^{1}(\Omega)\right) \Psi_{\mathrm{sc}^{-} f_{\text {hom }}^{q}}\left(\Omega, u_{z_{0}}+c, u\right) \\
\text { for every } \Omega^{\prime} \in \mathcal{A}_{0} \text { with } \Omega \subset \subset \Omega^{\prime} .
\end{gathered}
$$

In conclusion, once we observe that by $(2.2)$ it follows that

$$
\int_{\Omega^{\prime}} \operatorname{sc}^{-} f_{\text {hom }}^{q}(\nabla u) \mathrm{d} x+\int_{\Omega^{\prime}}\left(\mathrm{sc}^{-} f_{\text {hom }}^{q}\right)^{\infty}\left(\frac{\mathrm{d} D^{s} u}{\mathrm{~d}\left|D^{s} u\right|}\right) \mathrm{d}\left|D^{s} u\right|=\int_{\Omega} \mathrm{sc}^{-} f_{\text {hom }}^{q}(\nabla u) \mathrm{d} x+\left|\Omega^{\prime} \backslash \Omega\right| f_{\text {hom }}^{q}\left(z_{0}\right)
$$




$$
\begin{gathered}
+\int_{\Omega}\left(\mathrm{sc}^{-} f_{\text {hom }}^{q}\right)^{\infty}\left(\frac{\mathrm{d} D^{s} u}{\mathrm{~d}\left|D^{s} u\right|}\right) \mathrm{d}\left|D^{s} u\right|+\int_{\partial \Omega}\left(\mathrm{sc}^{-} f_{\mathrm{hom}}^{q}\right)^{\infty}\left(\left(u_{z_{0}}+c-u\right) \mathbf{n}_{\Omega}\right) \mathrm{d} \mathcal{H}^{n-1} \\
\text { for every } \Omega^{\prime} \in \mathcal{A}_{0} \text { with } \Omega \subset \subset \Omega^{\prime},
\end{gathered}
$$

and that $f_{\text {hom }}^{q}\left(z_{0}\right)<+\infty$ since $z_{0} \in C$ and (1.4) holds, the thesis comes from (5.4), (5.5), and (1.4) letting $\Omega^{\prime}$ decrease to $\Omega$.

Theorem 5.2. Let $f$ be as in (1.1), $q \in[1,+\infty], F_{0}^{\prime}$ and $F_{0}^{\prime \prime}$ be defined in (2.22), and $f_{\text {hom }}^{q}$ in (1.5). Let $C \subseteq \mathbf{R}^{n}$ be convex such that (1.2) $\div(1.4)$ hold. Then $f_{\text {hom }}^{q}$ is convex and

$$
\begin{gathered}
F_{0}^{\prime}\left(\Omega, \partial \Omega, u_{z_{0}}+c, u\right)=F_{0}^{\prime \prime}\left(\Omega, \partial \Omega, u_{z_{0}}+c, u\right) \\
=\int_{\Omega} \mathrm{sc}^{-} f_{\text {hom }}^{q}(\nabla u) \mathrm{d} x+\int_{\Omega}\left(\mathrm{sc}^{-} f_{\text {hom }}^{q}\right)^{\infty}\left(\frac{\mathrm{d} D^{s} u}{\mathrm{~d}\left|D^{s} u\right|}\right) \mathrm{d}\left|D^{s} u\right|+\int_{\partial \Omega}\left(\mathrm{sc}^{-} f_{\text {hom }}^{q}\right)^{\infty}\left(\left(u_{z_{0}}+c-u\right) \mathbf{n}_{\Omega}\right) \mathrm{d} \mathcal{H}^{n-1} \\
\text { for every } \Omega \in \mathcal{A}_{0} \text { convex, } z_{0} \in \operatorname{int}(C), c \in \mathbf{R}, u \in B V_{\mathrm{loc}}\left(\mathbf{R}^{n}\right) .
\end{gathered}
$$

Proof. First of all we prove that the assumptions of Theorem 2.8 are fulfilled by $g=\mathrm{sc}^{-} f_{\text {hom }}^{q}$.

It is clear that $\mathrm{sc}^{-} f_{\text {hom }}^{q}$, being lower semicontinuous, is also Borel. Moreover, the convexity of $\mathrm{sc}^{-} f_{\mathrm{hom}}^{q}$, Proposition 3.1 and (1.3) yield that $\operatorname{dom}\left(\mathrm{sc}^{-} f_{\text {hom }}^{q}\right)$ is convex and with nonempty interior. In addition, the continuity properties of $\mathrm{sc}^{-} f_{\text {hom }}^{q}$ imply the local boundedness condition in Theorem 2.8.

Finally, again by the convexity of $\mathrm{sc}^{-} f_{\text {hom }}^{q}$ we conclude that

$$
\mathrm{sc}^{-} f_{\mathrm{hom}}^{q}\left((1-t) z_{L}+t z\right) \leq(1-t) \mathrm{sc}^{-} f_{\mathrm{hom}}^{q}\left(z_{L}\right)+t \mathrm{sc}^{-} f_{\mathrm{hom}}^{q}(z) \leq \mathrm{sc}^{-} f_{\mathrm{hom}}^{q}(z)+(1-t) \mathrm{sc}^{-} f_{\mathrm{hom}}^{q}\left(z_{L}\right)
$$

for every bounded set $L \subseteq \operatorname{dom}\left(\mathrm{sc}^{-} f_{\mathrm{hom}}^{q}\right), z_{L} \in \operatorname{int}\left(\operatorname{dom}\left(\mathrm{sc}^{-} f_{\mathrm{hom}}^{q}\right)\right), z \in L, t \in[0,1]$,

from which also the last assumption of Theorem 2.8 follows.

The theorem now follows from Lemma 5.1, Theorem 2.8, and the obvious remark that sc $f_{\text {hom }}^{q}$, being convex and lower semicontinuous, agrees with $\left(\mathrm{sc}^{-} f_{\mathrm{hom}}^{q}\right)^{* *}$.

\section{The homogenization RESUlt For the CASE OF MiXed BOUNDARY CONDitions}

Let $f$ be as in (1.1), $\Omega \in \mathcal{A}_{0}, \Gamma \subseteq \partial \Omega, z_{0} \in \mathbf{R}^{n}, c \in \mathbf{R}$, and let $F_{0}^{\prime}\left(\Omega, \Gamma, u_{z_{0}}+c, \cdot\right)$ and $F_{0}^{\prime \prime}\left(\Omega, \Gamma, u_{z_{0}}+c, \cdot\right)$ be defined in (2.22). In this section we prove the identity between $F_{0}^{\prime}\left(\Omega, \Gamma, u_{z_{0}}+c, \cdot\right)$ and $F_{0}^{\prime \prime}\left(\Omega, \Gamma, u_{z_{0}}+c, \cdot\right)$, and an integral representation result for their common value.

We do this when $q=1$, and when (1.10) is fulfilled for some $\phi: \mathbf{R}^{n} \rightarrow[0,+\infty]$ convex with $\operatorname{int}(\operatorname{dom} \phi) \neq \emptyset$, $a \in L_{\text {loc }}^{1}\left(\mathbf{R}^{n}\right) Y$-periodic, and $M \geq 0$. We point out explicitly that (1.10) implies that $\operatorname{dom} f(x, \cdot)=\operatorname{dom} \phi$ for a.e. $x \in \mathbf{R}^{n}$.

Theorem 6.1. Let $f$ be as in (1.1), $F_{0}^{\prime}$ and $F_{0}^{\prime \prime}$ be defined in (2.22) with $q=1$, and $f_{\mathrm{hom}}^{1}$ in (1.5). Assume that (1.10) holds with $\phi: \mathbf{R}^{n} \rightarrow[0,+\infty]$ convex and satisfying $\operatorname{int}(\operatorname{dom} \phi) \neq \emptyset, a \in L_{\mathrm{loc}}^{1}\left(\mathbf{R}^{n}\right) Y$-periodic, and $M \geq 0$. Then $f_{\mathrm{hom}}^{1}$ is convex and

$$
F_{0}^{\prime}\left(\Omega, \Gamma, u_{z_{0}}+c, u\right)=F_{0}^{\prime \prime}\left(\Omega, \Gamma, u_{z_{0}}+c, u\right)=\int_{\Omega} \mathrm{sc}^{-} f_{\mathrm{hom}}^{1}(\nabla u) \mathrm{d} x
$$

for every $\Omega \in \mathcal{A}_{0}$ convex, $\Gamma \subseteq \partial \Omega, z_{0} \in \operatorname{int}(\operatorname{dom} \phi), c \in \mathbf{R}, u \in u_{z_{0}}+c+W_{0, \Gamma}^{1,1}(\Omega)$. 
Proof. Let $F^{\prime}, F^{\prime \prime}$ be given by (2.13) with $q=1$. Then it is clear that

$$
F_{-}^{\prime}(\Omega, u) \leq F_{0}^{\prime}\left(\Omega, \Gamma, u_{z_{0}}+c, u\right)
$$

for every $\Omega \in \mathcal{A}_{0}$ with Lipschitz boundary, $\Gamma \subseteq \partial \Omega, z_{0} \in \mathbf{R}^{n}, c \in \mathbf{R}, u \in L_{\text {loc }}^{1}\left(\mathbf{R}^{n}\right)$.

In order to treat the reverse inequality, let us first assume that

$$
0 \in \operatorname{int}(\operatorname{dom} \phi)
$$

and prove that

$$
F_{0}^{\prime \prime}(\Omega, \Gamma, c, u) \leq F^{\prime \prime}(\Omega, u)
$$

for every $\Omega \in \mathcal{A}_{0}$ with Lipschitz boundary, $\Gamma \subseteq \partial \Omega, u \in c+W_{0, \Gamma}^{1,1}(\Omega) \cap L^{\infty}(\Omega)$.

Let $\Omega, \Gamma, u$ be as in (6.3), and let us assume that $F^{\prime \prime}(\Omega, u)<+\infty$. Then by ii) of Lemma 4.2 , there exists $\left\{u_{h}\right\} \subseteq W_{\text {loc }}^{1,1}\left(\mathbf{R}^{n}\right)$ such that $u_{h} \rightarrow u$ in $L^{\infty}(\Omega)$, and

$$
\limsup _{h \rightarrow+\infty} \int_{\Omega} f\left(h x, \nabla u_{h}\right) \mathrm{d} x \leq F^{\prime \prime}(\Omega, u) .
$$

By (6.4), the left-hand side of (1.10), and the finiteness of $F^{\prime \prime}(\Omega, u)$, it follows that $\nabla u_{h}(x) \in$ dom $\phi$ for every $h \in \mathbf{N}$ sufficiently large, and a.e. $x \in \Omega$.

Let $B$ be an open set with $B \subset \subset \Omega$, moreover let $\psi \in C_{0}^{\infty}(\Omega)$ be such that $0 \leq \psi(x) \leq 1$ for every $x \in \Omega$, and $\psi(x)=1$ for every $x \in B$.

For every $h \in \mathbf{N}$, let $w_{h}$ be defined by $w_{h}=\psi u_{h}+(1-\psi) u$. Then obviously, $w_{h} \in c+W_{0, \Gamma}^{1,1}(\Omega)$ for every $h \in \mathbf{N}$, and $w_{h} \rightarrow u$ in $L^{\infty}(\Omega)$.

Let now $t \in[0,1[$. Then, by making use of the convexity of $f$, it results that

$$
\begin{aligned}
& \int_{\Omega} f\left(h x, t \nabla w_{h}\right) \mathrm{d} x \leq t \int_{\Omega} f\left(h x, \psi \nabla u_{h}+(1-\psi) \nabla u\right) \mathrm{d} x+(1-t) \int_{\Omega} f\left(h x, \frac{t}{1-t}\left(u_{h}-u\right) \nabla \psi\right) \mathrm{d} x \\
\leq & t \int_{\Omega} \psi(x) f\left(h x, \nabla u_{h}\right) \mathrm{d} x+t \int_{\Omega}(1-\psi(x)) f(h x, \nabla u) \mathrm{d} x+(1-t) \int_{\Omega} f\left(h x, \frac{t}{1-t}\left(u_{h}-u\right) \nabla \psi\right) \mathrm{d} x \\
\leq & \int_{\Omega} f\left(h x, \nabla u_{h}\right) \mathrm{d} x+\int_{\Omega \backslash B} f(h x, \nabla u) \mathrm{d} x+(1-t) \int_{\Omega} f\left(h x, \frac{t}{1-t}\left(u_{h}-u\right) \nabla \psi\right) \mathrm{d} x \text { for every } h \in \mathbf{N} .
\end{aligned}
$$

Hence, since clearly $t w_{h}+(1-t) c \rightarrow t u+(1-t) c$ in $L^{\infty}(\Omega)$, by virtue of $(6.4)$, we get that

$$
\begin{gathered}
F_{0}^{\prime \prime}(\Omega, \Gamma, c, t u+(1-t) c) \leq \limsup _{h \rightarrow+\infty} \int_{\Omega} f\left(h x, \nabla\left(t w_{h}+(1-t) c\right)\right) \mathrm{d} x=\limsup _{h \rightarrow+\infty} \int_{\Omega} f\left(h x, t \nabla w_{h}\right) \mathrm{d} x \\
\leq F^{\prime \prime}(\Omega, u)+\limsup _{h \rightarrow+\infty} \int_{\Omega \backslash B} f(h x, \nabla u) \mathrm{d} x+(1-t) \limsup _{h \rightarrow+\infty} \int_{\Omega} f\left(h x, \frac{t}{1-t}\left(u_{h}-u\right) \nabla \psi\right) \mathrm{d} x \\
\text { for every } t \in[0,1[.
\end{gathered}
$$

On the other hand, the finiteness of $F^{\prime \prime}(\Omega, u)$, and ii) of Lemma 4.1 yield that

$$
\limsup _{h \rightarrow+\infty} \int_{\Omega \backslash B} f(h x, \nabla u) \mathrm{d} x=\rho_{B}
$$

for some $\rho_{B} \in[0,+\infty[$, decreasing to 0 as $B$ increases to $\Omega$. 
Moreover, let us fix $r \in] 0$, dist $(0, \partial(\operatorname{dom} \phi))\left[\right.$. Then, since obviously $\left\|\left(u_{h}-u\right) \nabla \psi\right\|_{\left(L^{\infty}(\Omega)\right)^{n}} \rightarrow 0$, by using $(6.2)$ and the properties of $\psi$, one has that

for every $t \in\left[0,1\left[\right.\right.$ there exists $h_{t} \in \mathbf{N}$ such that

$$
\frac{t}{1-t}\left(u_{h}(x)-u(x)\right) \nabla \psi(x) \in \operatorname{dom} \phi \text { for a.e. } x \in \Omega \text { and every } h \in \mathbf{N} \cap\left[h_{t},+\infty[.\right.
$$

Consequently, once we denote by $\bar{z}_{1}, \ldots, \bar{z}_{2^{n}}$ the vertices of the cube centred in 0 and with sidelength $\frac{2 r}{\sqrt{n}}$, by (6.7), the convexity properties of $f$, and (1.10) it is easy to verify that

$$
\limsup _{h \rightarrow+\infty} \int_{\Omega} f\left(h x, \frac{t}{1-t}\left(u_{h}-u\right) \nabla \psi\right) \mathrm{d} x \leq|\Omega| \sum_{j=1}^{2^{n}} \int_{Y} f\left(y, \bar{z}_{j}\right) \mathrm{d} y<+\infty \text { for every } t \in[0,1[.
$$

Passing to the limit in (6.5) as $t$ increases to 1 , by (6.5), (6.6), (6.8), and (2.9), it follows that

$$
F_{0}^{\prime \prime}(\Omega, \Gamma, c, u) \leq \liminf _{t \rightarrow 1^{-}} F_{0}^{\prime \prime}(\Omega, \Gamma, c, t u+(1-t) c) \leq F^{\prime \prime}(\Omega, u)+\rho_{B} \text { for every } B \in \mathcal{A}_{0} \text { with } B \subset \subset \Omega,
$$

whence (6.3) follows by letting $B$ increase to $\Omega$.

Again under assumption (6.2), let us now prove that

$$
F_{0}^{\prime \prime}(\Omega, \Gamma, c, u) \leq F^{\prime \prime}(\Omega, u)
$$

for every $\Omega \in \mathcal{A}_{0}$ with Lipschitz boundary, $\Gamma \subseteq \partial \Omega, u \in c+W_{0, \Gamma}^{1,1}(\Omega)$.

To do this, let $\Omega, \Gamma, c, u$ be as in (6.9), and, for every $k \in \mathbf{N}$, let $T_{k} u$ be the truncation of $u$ at level $k$ defined by $T_{k} u=\min \{\max \{u,-k\}, k\}$.

It is clear that, since $u \in c+W_{0, \Gamma}^{1,1}(\Omega)$, then $T_{k} u \in c+W_{0, \Gamma}^{1,1}(\Omega) \cap L^{\infty}(\Omega)$ for every $k \in \mathbf{N}$ sufficiently large. Moreover

$$
\limsup _{k \rightarrow+\infty} F^{\prime \prime}\left(\Omega, T_{k} u\right) \leq F^{\prime \prime}(\Omega, u) .
$$

To show this, if $F^{\prime \prime}(\Omega, u)<+\infty$, let $\left\{u_{h}\right\} \subseteq W_{\text {loc }}^{1,1}\left(\mathbf{R}^{n}\right)$ be such that $u_{h} \rightarrow u$ in $L^{1}(\Omega)$, and

$$
F^{\prime \prime}(\Omega, u)=\limsup _{h \rightarrow+\infty} \int_{\Omega} f\left(h x, \nabla u_{h}\right) \mathrm{d} x .
$$

Then, for $k \in \mathbf{N}$,

$$
F^{\prime \prime}\left(\Omega, T_{k} u\right) \leq \limsup _{h \rightarrow+\infty} \int_{\Omega} f\left(h x, \nabla T_{k} u_{h}\right) \mathrm{d} x \leq F^{\prime \prime}(\Omega, u)+\limsup _{h \rightarrow+\infty} \int_{\left\{y \in \Omega:\left|u_{h}(y)\right| \geq k\right\}} f(h x, 0) \mathrm{d} x .
$$

Now it is clear that $\left|\left\{y \in \Omega:\left|u_{h}(y)\right| \geq k\right\}\right| \leq \frac{1}{k} \int_{\Omega}\left|u_{h}\right| \mathrm{d} x$ for every $h \in \mathbf{N}$. Consequently, by (6.2), (1.10), and the equi-absolute continuity of the integrals $\int . f(h x, 0) \mathrm{d} x$, it turns out that

$$
\limsup _{k \rightarrow+\infty} \limsup _{h \rightarrow+\infty} \int_{\left\{y \in \Omega:\left|u_{h}(y)\right| \geq k\right\}} f(h x, 0) \mathrm{d} x=0,
$$

from which (6.10) follows.

By (2.9), (6.3), and (6.10), inequality (6.9) follows once we observe that $T_{k} u \rightarrow u$ in $L^{1}(\Omega)$.

In conclusion, if (6.2) is dropped, for $z_{0} \in \operatorname{int}(\operatorname{dom} \phi)$, we only have to apply (6.3) with $f$ replaced by $(x, z) \in \mathbf{R}^{n} \times \mathbf{R}^{n} \mapsto f\left(x, z_{0}+z\right)$, to get

$$
F_{0}^{\prime \prime}\left(\Omega, \Gamma, u_{z_{0}}+c, u\right) \leq F^{\prime \prime}(\Omega, u)
$$


for every $\Omega \in \mathcal{A}_{0}$ with Lipschitz boundary, $\Gamma \subseteq \partial \Omega, z_{0} \in \operatorname{int}(\operatorname{dom} \phi), c \in \mathbf{R}, u \in u_{z_{0}}+c+W_{0, \Gamma}^{1,1}(\Omega)$.

By (6.1), (6.11), and Theorem 4.4 the thesis follows.

\section{Applications to minimum problems}

In this section we obtain some homogenization results for minima and minimizers of some classes of variational problems for energies of integral type both in $B V$ and Sobolev spaces.

The choice of the space framework clearly depends on the coerciveness properties of the energy densities $f$. Thus we take $p \in[1,+\infty]$, and consider the following coerciveness assumptions

$$
\begin{cases}c_{1}|z|^{p}-c_{2} \leq f(x, z) \text { for a.e. } x \in \mathbf{R}^{n}, \text { and every } z \in \mathbf{R}^{n} & \text { if } p \in[1,+\infty[ \\ \operatorname{dom} f(x, \cdot) \subseteq\left\{z \in \mathbf{R}^{n}:|z| \leq R\right\} \text { for a.e. } x \in \mathbf{R}^{n} & \text { if } p=+\infty\end{cases}
$$

for some $c_{1}>0, c_{2} \in \mathbf{R}$, and $R>0$.

If for every $q \in[1,+\infty], f_{\text {hom }}^{q}$ is given by (1.5), and if (7.1) is fulfilled, then the corresponding coerciveness properties of $f_{\text {hom }}^{q}$ are given by

$$
\begin{cases}c_{1}|z|^{p}-c_{2} \leq f_{\text {hom }}^{q}(z) \text { for every } z \in \mathbf{R}^{n} & \text { if } p \in[1,+\infty[ \\ \operatorname{dom} f_{\text {hom }}^{q} \subseteq\left\{z \in \mathbf{R}^{n}:|z| \leq R\right\} & \text { if } p=+\infty\end{cases}
$$

as described in the following result.

Proposition 7.1. Let $f$ be as in (1.1), $q \in[1,+\infty]$, and $f_{\text {hom }}^{q}$ be defined in (1.5). Assume that (7.1) is fulfilled for some $p \in[1,+\infty]$. Then (7.2) holds.

Proof. The result follows from i) of Proposition 3.3 applied with $\phi=c_{1}|\cdot|^{p}-c_{2}$ if $p \in[1,+\infty[$, or with $\phi$ equal to the indicator function of $\left\{z \in \mathbf{R}^{n}:|z| \leq R\right\}$ if $p=+\infty$.

Set, for every $p \in[1,+\infty]$,

$$
p^{\prime}=\left\{\begin{array}{ll}
+\infty & \text { if } p=1 \\
\frac{p}{p-1} & \text { if } 1<p<+\infty \\
1 & \text { if } p=+\infty
\end{array} \quad p^{*}= \begin{cases}\frac{n p}{n-p} & \text { if } p<n \\
+\infty & \text { otherwise. }\end{cases}\right.
$$

We start with the case of Neumann minimum problems in $B V$ spaces.

Theorem 7.2. Let $f$ be as in (1.1) and satisfy (7.1) with $p=1$, let $q \in[1,+\infty]$, and let $f_{\text {hom }}^{q}$ be defined in (1.5). Let $C \subseteq \mathbf{R}^{n}$ be convex such that (1.2) $\div(1.4)$ hold. For every $h \in \mathbf{N}, \Omega \in \mathcal{A}_{0}$ convex, $\left.\lambda \in\right] 0,+\infty[$, $r \in] 1,1^{*}\left[, \beta \in L^{\infty}(\Omega)\right.$ let

$$
\begin{aligned}
i_{h}^{N}(q, \Omega, \lambda, r, \beta)= & \inf \left\{\int_{\Omega} f(h x, \nabla u) \mathrm{d} x+\lambda \int_{\Omega}|u|^{r} \mathrm{~d} x+\int_{\Omega} \beta u \mathrm{~d} x: u \in W^{1, q}(\Omega)\right\}, \\
m_{\infty}^{N}(q, \Omega, \lambda, r, \beta)= & \min \left\{\int_{\Omega} \operatorname{sc}^{-} f_{\text {hom }}^{q}(\nabla u) \mathrm{d} x+\int_{\Omega}\left(\operatorname{sc}^{-} f_{\text {hom }}^{q}\right)^{\infty}\left(\frac{\mathrm{d} D^{s} u}{\mathrm{~d}\left|D^{s} u\right|}\right) \mathrm{d}\left|D^{s} u\right|\right. \\
& \left.+\lambda \int_{\Omega}|u|^{r} \mathrm{~d} x+\int_{\Omega} \beta u \mathrm{~d} x: u \in B V(\Omega)\right\},
\end{aligned}
$$

and let $\left\{\tilde{u}_{h}\right\} \subseteq W^{1, q}(\Omega)$ be such that

$$
\lim _{h \rightarrow+\infty}\left(\int_{\Omega} f\left(h x, \nabla \tilde{u}_{h}\right) \mathrm{d} x+\lambda \int_{\Omega}\left|\tilde{u}_{h}\right|^{r} \mathrm{~d} x+\int_{\Omega} \beta \tilde{u}_{h} \mathrm{~d} x-i_{h}^{N}(q, \Omega, \lambda, r, \beta)\right)=0 .
$$


Then $f_{\mathrm{hom}}^{q}$ is convex and satisfies (7.2) with $p=1,\left\{i_{h}^{N}(q, \Omega, \lambda, r, \beta)\right\}$ converges to $m_{\infty}^{N}(q, \Omega, \lambda, r, \beta),\left\{\tilde{u}_{h}\right\}$ is compact in $L^{1}(\Omega)$, and its converging subsequences converge to solutions of $m_{\infty}^{N}(q, \Omega, \lambda, r, \beta)$.

Proof. The properties of $f_{\text {hom }}^{q}$ follow from Proposition 3.1 and Proposition 7.1.

Let $\Omega, \lambda, r, \beta$ be as above, and let us preliminarily prove that

$$
\begin{gathered}
\Gamma^{-}\left(L^{1}(\Omega)\right) \lim _{h \rightarrow+\infty}\left\{F_{h}(\Omega, u)+\lambda \int_{\Omega}|u|^{r} \mathrm{~d} x\right\} \\
= \begin{cases}\int_{\Omega} \mathrm{sc}^{-} f_{\text {hom }}^{q}(\nabla u) \mathrm{d} x+\int_{\Omega}\left(\mathrm{sc}^{-} f_{\text {hom }}^{q}\right)^{\infty}\left(\frac{\mathrm{d} D^{s} u}{\mathrm{~d}\left|D^{s} u\right|}\right) \mathrm{d}\left|D^{s} u\right|+\lambda \int_{\Omega}|u|^{r} \mathrm{~d} x & \text { if } u \in B V(\Omega) \\
+\infty & \text { if } u \in L_{\text {loc }}^{1}\left(\mathbf{R}^{n}\right) \backslash B V(\Omega)\end{cases}
\end{gathered}
$$

$$
\text { for every } u \in L_{\text {loc }}^{1}\left(\mathbf{R}^{n}\right) \text {, }
$$

where, for every $h \in \mathbf{N}, F_{h}(\Omega, \cdot)$ is defined by $(2.12)$.

To this aim, we take $u \in B V(\Omega)$ such that $\int_{\Omega} \mathrm{sc}^{-} f_{\text {hom }}^{q}(\nabla u) \mathrm{d} x+\int_{\Omega}\left(\mathrm{sc}^{-} f_{\text {hom }}^{q}\right)^{\infty}\left(\frac{\mathrm{d} D^{s} u}{\mathrm{~d}\left|D^{s} u\right|}\right) \mathrm{d}\left|D^{s} u\right|+\lambda \int_{\Omega}|u|^{r} \mathrm{~d} x<$ $+\infty$, and observe that, because of the Lipschitz regularity of $\partial \Omega$ and of the extension properties of $B V(\Omega)$ functions, it is not restrictive to assume that $u \in B V_{\mathrm{loc}}\left(\mathbf{R}^{n}\right)$. Then, Theorem 4.4 provides $\left\{u_{h}\right\} \subseteq W_{\mathrm{loc}}^{1, q}\left(\mathbf{R}^{n}\right)$ such that $u_{h} \rightarrow u$ in $L^{1}(\Omega)$, and

$$
\int_{\Omega} \mathrm{sc}^{-} f_{\text {hom }}^{q}(\nabla u) \mathrm{d} x+\int_{\Omega}\left(\mathrm{sc}^{-} f_{\text {hom }}^{q}\right)^{\infty}\left(\frac{\mathrm{d} D^{s} u}{\mathrm{~d}\left|D^{s} u\right|}\right) \mathrm{d}\left|D^{s} u\right|=\limsup _{h \rightarrow+\infty} \int_{\Omega} f\left(h x, \nabla u_{h}\right) \mathrm{d} x .
$$

Now, by (7.1) with $p=1$, the Lipschitz regularity of $\partial \Omega$, and the compact embedding of $B V(\Omega)$ in $L^{r}(\Omega)$, it follows that $u_{h} \rightarrow u$ in $L^{r}(\Omega)$, and therefore

$$
\begin{aligned}
& \Gamma^{-}\left(L^{1}(\Omega)\right) \limsup _{h \rightarrow+\infty}\left\{F_{h}(\Omega, u)+\lambda \int_{\Omega}|u|^{r} \mathrm{~d} x\right\} \leq \limsup _{h \rightarrow+\infty}\left\{\int_{\Omega} f\left(h x, \nabla u_{h}\right) \mathrm{d} x+\lambda \int_{\Omega}\left|u_{h}\right|^{r} \mathrm{~d} x\right\} \\
= & \int_{\Omega} \mathrm{sc}^{-} f_{\text {hom }}^{q}(\nabla u) \mathrm{d} x+\int_{\Omega}\left(\mathrm{sc}^{-} f_{\text {hom }}^{q}\right)^{\infty}\left(\frac{\mathrm{d} D^{s} u}{\mathrm{~d}\left|D^{s} u\right|}\right) \mathrm{d}\left|D^{s} u\right|+\lambda \int_{\Omega}|u|^{r} \mathrm{~d} x \text { for every } u \in B V(\Omega) .
\end{aligned}
$$

On the other side, if $u \in L_{\text {loc }}^{1}\left(\mathbf{R}^{n}\right)$ satisfies $\Gamma^{-}\left(L^{1}(\Omega)\right) \liminf _{h \rightarrow+\infty}\left\{F_{h}(\Omega, u)+\lambda \int_{\Omega}|u|^{r} \mathrm{~d} x\right\}<+\infty$, there exists $\left\{u_{h}\right\} \subseteq L_{\text {loc }}^{1}\left(\mathbf{R}^{n}\right)$ and $\left\{h_{k}\right\} \subseteq \mathbf{N}$ strictly increasing, such that $u_{h} \rightarrow u$ in $L^{1}(\Omega),\left\{u_{h_{k}}\right\} \subseteq W_{\text {loc }}^{1, q}\left(\mathbf{R}^{n}\right)$, and

$$
\Gamma^{-}\left(L^{1}(\Omega)\right) \liminf _{h \rightarrow+\infty}\left\{F_{h}(\Omega, u)+\lambda \int_{\Omega}|u|^{r} \mathrm{~d} x\right\}=\liminf _{k \rightarrow+\infty}\left\{\int_{\Omega} f\left(h_{k} x, \nabla u_{h_{k}}\right) \mathrm{d} x+\lambda \int_{\Omega}\left|u_{h_{k}}\right|^{r} \mathrm{~d} x\right\} .
$$

Then, the same coerciveness and compactness arguments as above, provide that $u \in B V(\Omega)$ and that $u_{h_{k}} \rightarrow u$ in $L^{r}(\Omega)$. Consequently, (7.5) and Theorem 4.4 imply that

$$
\begin{gathered}
\Gamma^{-}\left(L^{1}(\Omega)\right) \liminf _{h \rightarrow+\infty}\left\{F_{h}(\Omega, u)+\lambda \int_{\Omega}|u|^{r} \mathrm{~d} x\right\} \geq \liminf _{k \rightarrow+\infty} \int_{\Omega} f\left(h_{k} x, \nabla u_{h_{k}}\right) \mathrm{d} x+\lambda \int_{\Omega}|u|^{r} \mathrm{~d} x \\
\geq \liminf _{h \rightarrow+\infty} F_{h}\left(\Omega, u_{h}\right)+\lambda \int_{\Omega}|u|^{r} \mathrm{~d} x \\
\geq \begin{cases}\int_{\Omega} \mathrm{sc}^{-} f_{\text {hom }}^{q}(\nabla u) \mathrm{d} x+\int_{\Omega}\left(\mathrm{sc}^{-} f_{\mathrm{hom}}^{q}\right)^{\infty}\left(\frac{\mathrm{d} D^{s} u}{\mathrm{~d}\left|D^{s} u\right|}\right) \mathrm{d}\left|D^{s} u\right|+\lambda \int_{\Omega}|u|^{r} \mathrm{~d} x & \text { if } u \in B V(\Omega) \\
+\infty & \text { if } u \in L_{\text {loc }}^{1}\left(\mathbf{R}^{n}\right) \backslash B V(\Omega)\end{cases}
\end{gathered}
$$

for every $u \in L_{\text {loc }}^{1}\left(\mathbf{R}^{n}\right)$.

By (7.4) and (7.6), equality (7.3) follows easily.

Now, observe that (7.1) with $p=1$ ensures that the functionals $u \in L_{\text {loc }}^{1}\left(\mathbf{R}^{n}\right) \mapsto F_{h}(\Omega, u)+\lambda \int_{\Omega}|u|^{r} \mathrm{~d} x+$ $\int_{\Omega} \beta u \mathrm{~d} x$ are equicoercive once $L_{\text {loc }}^{1}\left(\mathbf{R}^{n}\right)$ is equipped with the $L^{1}(\Omega)$-topology. 
Therefore the theorem follows from (7.3), Theorem 2.7, and by the $L^{1}(\Omega)$-continuity of the functional $u \in$ $L_{\mathrm{loc}}^{1}\left(\mathbf{R}^{n}\right) \mapsto \int_{\Omega} \beta u \mathrm{~d} x$

We now treat the case of Neumann minimum problems in Sobolev spaces.

Theorem 7.3. Let $f$ be as in (1.1) and satisfy (7.1), let $p \in] 1,+\infty], q \in[p,+\infty]$, and let $f_{\text {hom }}^{q}$ be defined in (1.5). Let $C \subseteq \mathbf{R}^{n}$ be convex such that (1.2) $\div(1.4)$ hold. For every $h \in \mathbf{N}, \Omega \in \mathcal{A}_{0}$ convex, $\left.\lambda \in\right] 0,+\infty[$, $r \in] 1, p^{*}\left[, \beta \in L^{p^{\prime}}(\Omega), \gamma \in L^{p^{\prime}}(\partial \Omega)\right.$ let

$$
\begin{aligned}
& i_{h}^{N}(q, \Omega, \lambda, r, \beta, \gamma)=\inf \left\{\int_{\Omega} f(h x, \nabla u) \mathrm{d} x+\lambda \int_{\Omega}|u|^{r} \mathrm{~d} x+\int_{\Omega} \beta u \mathrm{~d} x+\int_{\partial \Omega} \gamma u \mathrm{~d} \mathcal{H}^{n-1}: u \in W^{1, q}(\Omega)\right\} \\
& m_{\infty}^{N}(q, \Omega, \lambda, r, \beta, \gamma)=\min \left\{\int_{\Omega} \mathrm{sc}^{-} f_{\mathrm{hom}}^{q}(\nabla u) \mathrm{d} x+\lambda \int_{\Omega}|u|^{r} \mathrm{~d} x+\int_{\Omega} \beta u \mathrm{~d} x+\int_{\partial \Omega} \gamma u \mathrm{~d} \mathcal{H}^{n-1}: u \in W^{1, p}(\Omega)\right\},
\end{aligned}
$$

and let $\left\{\tilde{u}_{h}\right\} \subseteq W^{1, q}(\Omega)$ be such that

$$
\lim _{h \rightarrow+\infty}\left(\int_{\Omega} f\left(h x, \nabla \tilde{u}_{h}\right) \mathrm{d} x+\lambda \int_{\Omega}\left|\tilde{u}_{h}\right|^{r} \mathrm{~d} x+\int_{\Omega} \beta \tilde{u}_{h} \mathrm{~d} x+\int_{\partial \Omega} \gamma \tilde{u}_{h} \mathrm{~d} \mathcal{H}^{n-1}-i_{h}^{N}(q, \Omega, \lambda, r, \beta, \gamma)\right)=0 .
$$

Then $f_{\text {hom }}^{q}$ is convex and satisfies (7.2), $\left\{i_{h}^{N}(q, \Omega, \lambda, r, \beta, \gamma)\right\}$ converges to $m_{\infty}^{N}(q, \Omega, \lambda, r, \beta, \gamma),\left\{\tilde{u}_{h}\right\}$ is compact in $L^{p}(\Omega)$, and its converging subsequences converge to solutions of $m_{\infty}^{N}(q, \Omega, \lambda, r, \beta, \gamma)$.

Moreover, if $q=p$ and (1.9) too holds, then $\mathrm{sc}^{-} f_{\text {hom }}^{p}=f_{\text {hom }}^{p}$, for every $z \in \mathbf{R}^{n}$ the infimum in the definition of $f_{\text {hom }}^{p}(z)$ is attained, problems in (7.7) have solutions, and for every $h \in \mathbf{N}$ one can take $\tilde{u}_{h}$ as a minimizer of $i_{h}^{N}(q, \Omega, \lambda, r, \beta, \gamma)$.

Proof. The properties of $f_{\text {hom }}^{q}$ follow from Proposition 3.1, Proposition 7.1, and Proposition 3.4.

Let $\Omega, \lambda, r, \beta, \gamma$ be as above, and let $P(\Omega, \cdot)$ be defined by

$$
P(\Omega, \cdot): u \in L_{\text {loc }}^{1}\left(\mathbf{R}^{n}\right) \mapsto \begin{cases}\lambda \int_{\Omega}|u|^{r} \mathrm{~d} x+\int_{\Omega} \beta u \mathrm{~d} x+\int_{\partial \Omega} \gamma u \mathrm{~d} \mathcal{H}^{n-1} & \text { if } u \in W^{1, p}(\Omega) \\ +\infty & \text { if } u \in L_{\mathrm{loc}}^{1}\left(\mathbf{R}^{n}\right) \backslash W^{1, p}(\Omega) .\end{cases}
$$

Let us prove that

$$
= \begin{cases}\int_{\Omega} \mathrm{sc}^{-} f_{\text {hom }}^{q}(\nabla u) \mathrm{d} x+\lambda \int_{\Omega}|u|^{r} \mathrm{~d} x+\int_{\Omega} \beta u \mathrm{~d} x+\int_{\partial \Omega} \gamma u \mathrm{~d} \mathcal{H}^{n-1} & \text { if } u \in W^{1, p}(\Omega) \\ +\infty & \text { if } u \in L_{\text {loc }}^{1}\left(\mathbf{R}^{n}\right) \backslash W^{1, p}(\Omega)\end{cases}
$$

for every $u \in L_{\text {loc }}^{1}\left(\mathbf{R}^{n}\right)$,

where, for every $h \in \mathbf{N}, F_{h}(\Omega, \cdot)$ is defined by $(2.12)$.

To do this, we take $u \in W^{1, p}(\Omega)$ such that $\int_{\Omega} \mathrm{sc}^{-} f_{\text {hom }}^{q}(\nabla u) \mathrm{d} x+\lambda \int_{\Omega}|u|^{r} \mathrm{~d} x+\int_{\Omega} \beta u \mathrm{~d} x+\int_{\partial \Omega} \gamma u \mathrm{~d} \mathcal{H}^{n-1}<+\infty$. Then, Theorem 4.4 provides $\left\{u_{h}\right\} \subseteq W_{\text {loc }}^{1, q}\left(\mathbf{R}^{n}\right)$ such that $u_{h} \rightarrow u$ in $L^{1}(\Omega)$, and

$$
\int_{\Omega} \mathrm{sc}^{-} f_{\text {hom }}^{q}(\nabla u) \mathrm{d} x=\limsup _{h \rightarrow+\infty} \int_{\Omega} f\left(h x, \nabla u_{h}\right) \mathrm{d} x
$$

Now, by (7.1), the Lipschitz regularity of $\partial \Omega$, and the Rellich-Kondrachov Compactness Theorem, it turns out that $u_{h} \rightarrow u$ in $L^{r}(\Omega)$ and in weak- $W^{1, p}(\Omega)$ if $\left.p \in\right] 1,+\infty\left[\right.$, or in $L^{\infty}(\Omega)$ and in weak ${ }^{*} W^{1, \infty}(\Omega)$ if $p=+\infty$. Therefore, by taking into account the continuity of $v \in W^{1, p}(\Omega) \mapsto \int_{\Omega} \beta u \mathrm{~d} x+\int_{\partial \Omega} \gamma v \mathrm{~d} \mathcal{H}^{n-1}$ with respect to the weak- $W^{1, p}(\Omega)$-topology if $\left.p \in\right] 1,+\infty\left[\right.$, or the weak ${ }^{*} W^{1, \infty}(\Omega)$ one if $p=+\infty$, we conclude that

$$
\begin{gathered}
\Gamma^{-}\left(L^{1}(\Omega)\right) \limsup _{h \rightarrow+\infty}\left\{F_{h}(\Omega, u)+P(\Omega, u)\right\} \\
\leq \limsup _{h \rightarrow+\infty}\left\{\int_{\Omega} f\left(h x, \nabla u_{h}\right) \mathrm{d} x+\lambda \int_{\Omega}\left|u_{h}\right|^{r} \mathrm{~d} x+\int_{\Omega} \beta u_{h} \mathrm{~d} x+\int_{\partial \Omega} \gamma u_{h} \mathrm{~d} \mathcal{H}^{n-1}\right\}
\end{gathered}
$$




$$
=\int_{\Omega} \mathrm{sc}^{-} f_{\text {hom }}^{q}(\nabla u) \mathrm{d} x+\lambda \int_{\Omega}|u|^{r} \mathrm{~d} x+\int_{\Omega} \beta u \mathrm{~d} x+\int_{\partial \Omega} \gamma u \mathrm{~d} \mathcal{H}^{n-1} \text { for every } u \in W^{1, p}(\Omega) .
$$

On the other side, if $u \in L_{\text {loc }}^{1}\left(\mathbf{R}^{n}\right)$ satisfies $\Gamma^{-}\left(L^{1}(\Omega)\right) \liminf _{h \rightarrow+\infty}\left\{F_{h}(\Omega, u)+P(\Omega, u)\right\}<+\infty$, there exists $\left\{u_{h}\right\} \subseteq L_{\mathrm{loc}}^{1}\left(\mathbf{R}^{n}\right)$ and $\left\{h_{k}\right\} \subseteq \mathbf{N}$ strictly increasing, such that $u_{h} \rightarrow u$ in $L^{1}(\Omega),\left\{u_{h_{k}}\right\} \subseteq W_{\mathrm{loc}}^{1, q}\left(\mathbf{R}^{n}\right)$, and

$$
\begin{gathered}
\Gamma^{-}\left(L^{1}(\Omega)\right) \liminf _{h \rightarrow+\infty}\left\{F_{h}(\Omega, u)+P(\Omega, u)\right\} \\
=\liminf _{k \rightarrow+\infty}\left\{\int_{\Omega} f\left(h_{k} x, \nabla u_{h_{k}}\right) \mathrm{d} x+\lambda \int_{\Omega}\left|u_{h_{k}}\right|^{r} \mathrm{~d} x+\int_{\Omega} \beta u_{h_{k}} \mathrm{~d} x+\int_{\partial \Omega} \gamma u_{h_{k}} \mathrm{~d} \mathcal{H}^{n-1}\right\} .
\end{gathered}
$$

Then, the same coerciveness arguments as above, provide that $u \in W^{1, p}(\Omega)$ and that $u_{h_{k}} \rightarrow u$ in $L^{r}(\Omega)$ and in weak- $W^{1, p}(\Omega)$ if $\left.p \in\right] 1,+\infty$ [, or in $L^{\infty}(\Omega)$ and in weak ${ }^{*} W^{1, \infty}(\Omega)$ if $p=+\infty$. Consequently, (7.10), the above continuity arguments, and Theorem 4.4 imply that

$$
\begin{gathered}
\Gamma^{-}\left(L^{1}(\Omega)\right) \liminf _{h \rightarrow+\infty}\left\{F_{h}(\Omega, u)+P(\Omega, u)\right\} \\
\geq \liminf _{k \rightarrow+\infty} \int_{\Omega} f\left(h_{k} x, \nabla u_{h_{k}}\right) \mathrm{d} x+\liminf _{h \rightarrow+\infty}\left\{\lambda \int_{\Omega}\left|u_{h_{k}}\right|^{r} \mathrm{~d} x+\int_{\Omega} \beta u_{h_{k}} \mathrm{~d} x+\int_{\partial \Omega} \gamma u_{h_{k}} \mathrm{~d} \mathcal{H}^{n-1}\right\} \\
\geq \liminf _{h \rightarrow+\infty} F_{h}\left(\Omega, u_{h}\right)+\lambda \int_{\Omega}|u|^{r} \mathrm{~d} x+\int_{\Omega} \beta u \mathrm{~d} x+\int_{\partial \Omega} \gamma u \mathrm{~d} \mathcal{H}^{n-1} \\
\geq \begin{cases}\int_{\Omega} \mathrm{sc}^{-} f_{\text {hom }}^{q}(\nabla u) \mathrm{d} x+\lambda \int_{\Omega}|u|^{r} \mathrm{~d} x+\int_{\Omega} \beta u \mathrm{~d} x+\int_{\partial \Omega} \gamma u \mathrm{~d} \mathcal{H}^{n-1} & \text { if } u \in W^{1, p}(\Omega) \\
+\infty & \text { if } u \in L_{\mathrm{loc}}^{1}\left(\mathbf{R}^{n}\right) \backslash W^{1, p}(\Omega)\end{cases}
\end{gathered}
$$

By (7.9) and (7.11), equality (7.8) follows.

for every $u \in L_{\text {loc }}^{1}\left(\mathbf{R}^{n}\right)$.

We now observe that (7.1) ensures that the functionals $u \in L_{\text {loc }}^{1}\left(\mathbf{R}^{n}\right) \mapsto F_{h}(\Omega, u)+P(\Omega, u)$ are equicoercive once $L_{\text {loc }}^{1}\left(\mathbf{R}^{n}\right)$ is equipped with the $L^{1}(\Omega)$-topology.

In fact, when $p<+\infty$, by (7.1), Hölder Inequality, and Sobolev Imbedding Theorem it is easy to see that there exists $\left.C_{\Omega} \in\right] 0,+\infty\left[\right.$ such that (we denote here by $C_{\Omega}$ various constants depending only on $\Omega$ )

$$
\begin{gathered}
F_{h}(\Omega, u)+P(\Omega, u) \geq c_{1} \int_{\Omega}|\nabla u|^{p} \mathrm{~d} x+\lambda \int_{\Omega}|u|^{r} \mathrm{~d} x-\|\beta\|_{L^{p^{\prime}}(\Omega)}\|u\|_{L^{p}(\Omega)}-\|\gamma\|_{L^{p^{\prime}}(\partial \Omega)}\|u\|_{L^{p}(\partial \Omega)}-c_{2}|\Omega| \\
\geq c_{1}\|\nabla u\|_{\left(L^{p}(\Omega)\right)^{n}}^{p}+\lambda\|u\|_{L^{r}(\Omega)}^{r}-C_{\Omega}\left(\|\beta\|_{L^{p^{\prime}}(\Omega)}+\|\gamma\|_{L^{p^{\prime}(\partial \Omega)}}\right)\|u\|_{W^{1, p}(\Omega)}-c_{2}|\Omega| \\
\geq c_{1}\|\nabla u\|_{\left(L^{p}(\Omega)\right)^{n}}^{p}+\lambda\|u\|_{L^{r}(\Omega)}^{r}-C_{\Omega}\left(\|\beta\|_{L^{p^{\prime}}(\Omega)}+\|\gamma\|_{L^{p^{\prime}}(\partial \Omega)}\right)\left(\|u\|_{L^{r}(\Omega)}+\|\nabla u\|_{\left(L^{p}(\Omega)\right)^{n}}\right)-c_{2}|\Omega| \\
\text { for every } h \in \mathbf{N}, u \in W_{\text {loc }}^{1, q}(\Omega),
\end{gathered}
$$

or, when $p=+\infty$,

$$
\begin{gathered}
F_{h}(\Omega, u)+P(\Omega, u) \geq \lambda\|u\|_{L^{r}(\Omega)}^{r}-\|\beta\|_{L^{1}(\Omega)}\|u\|_{L^{\infty}(\Omega)}-\|\gamma\|_{L^{1}(\partial \Omega)}\|u\|_{L^{\infty}(\partial \Omega)} \\
\geq \lambda\|u\|_{L^{r}(\Omega)}^{r}-\left(\|\beta\|_{L^{1}(\Omega)}+\|\gamma\|_{L^{1}(\partial \Omega)}\right)\|u\|_{W^{1, \infty}(\Omega)} \\
\geq \lambda\|u\|_{L^{r}(\Omega)}^{r}-C_{\Omega}\left(\|\beta\|_{L^{1}(\Omega)}+\|\gamma\|_{L^{1}(\partial \Omega)}\right)\left(\|u\|_{L^{r}(\Omega)}+R\right) \text { for every } h \in \mathbf{N}, u \in W_{\text {loc }}^{1, \infty}\left(\mathbf{R}^{n}\right),
\end{gathered}
$$

from which, together with the Rellich-Kondrachov Compactness Theorem, the desired equicoerciveness follows.

Therefore (7.8) and Theorem 2.7 (applied with $E \equiv 0$ ) imply that $\left\{i_{h}^{N}(q, \Omega, \lambda, r, \beta, \gamma)\right\}$ converges to $m_{\infty}^{N}(q, \Omega, \lambda, r, \beta, \gamma)$, that $\left\{\tilde{u}_{h}\right\}$ is compact in $L^{1}(\Omega)$, and that its converging subsequences converge to solutions of $m_{\infty}^{N}(q, \Omega, \lambda, r, \beta, \gamma)$.

This completes the proof, once we observe that (7.12), (7.13) and the Rellich-Kondrachov Compactness Theorem entail the compactness in $L^{p}(\Omega)$ of $\left\{\tilde{u}_{h}\right\}$. 
Finally, if $q=p$ and if (1.9) holds too, the existence of the solutions of the problems in (7.7) follows from the above coerciveness arguments and Theorem 2.5.

We now pass to the case of Dirichlet minimum problems. We start with the one in $B V$ spaces.

Theorem 7.4. Let $f$ be as in (1.1) and satisfy (7.1) with $p=1$, let $q \in[1,+\infty]$, and let $f_{\text {hom }}^{q}$ be defined in (1.5). Let $C \subseteq \mathbf{R}^{n}$ be convex such that (1.2) $\div(1.4)$ hold. For every $h \in \mathbf{N}, \Omega \in \mathcal{A}_{0}$ convex, $\left.\lambda \in\right] 0,+\infty[$, $r \in] 1,1^{*}\left[, \beta \in L^{\infty}(\Omega), z_{0} \in \operatorname{int}(C), c \in \mathbf{R}\right.$ let

$$
\begin{gathered}
i_{h}^{D}(q, \Omega, \lambda, r, \beta)=\inf \left\{\int_{\Omega} f(h x, \nabla u) \mathrm{d} x+\lambda \int_{\Omega}|u|^{r} \mathrm{~d} x+\int_{\Omega} \beta u \mathrm{~d} x: u \in u_{z_{0}}+c+W_{0}^{1, q}(\Omega)\right\}, \\
m_{\infty}^{D}(q, \Omega, \lambda, r, \beta)=\min \left\{\int_{\Omega} \mathrm{sc}^{-} f_{\text {hom }}^{q}(\nabla u) \mathrm{d} x+\int_{\Omega}\left(\operatorname{sc}^{-} f_{\text {hom }}^{q}\right)^{\infty}\left(\frac{\mathrm{d} D^{s} u}{\mathrm{~d}\left|D^{s} u\right|}\right) \mathrm{d}\left|D^{s} u\right|\right. \\
\left.+\int_{\partial \Omega}\left(\operatorname{sc}^{-} f_{\text {hom }}^{q}\right)^{\infty}\left(\left(u-u_{z_{0}}-c\right) \mathbf{n}_{\Omega}\right) \mathrm{d} \mathcal{H}^{n-1}+\lambda \int_{\Omega}|u|^{r} \mathrm{~d} x+\int_{\Omega} \beta u \mathrm{~d} x: u \in B V(\Omega)\right\},
\end{gathered}
$$

and let $\left\{\tilde{u}_{h}\right\} \subseteq u_{z_{0}}+c+W_{0}^{1, q}(\Omega)$ be such that

$$
\lim _{h \rightarrow+\infty}\left(\int_{\Omega} f\left(h x, \nabla \tilde{u}_{h}\right) \mathrm{d} x+\lambda \int_{\Omega}|u|^{r} \mathrm{~d} x+\int_{\Omega} \beta \tilde{u}_{h} \mathrm{~d} x-i_{h}^{D}(q, \Omega, \lambda, r, \beta)\right)=0 .
$$

Then $f_{\text {hom }}^{q}$ is convex and satisfies (7.2) with $p=1,\left\{i_{h}^{D}(q, \Omega, \lambda, r, \beta)\right\}$ converges to $m_{\infty}^{D}(q, \Omega, \lambda, r, \beta),\left\{\tilde{u}_{h}\right\}$ is compact in $L^{1}(\Omega)$, and its converging subsequences converge to solutions of $m_{\infty}^{D}(q, \Omega, \lambda, r, \beta)$.

Proof. The properties of $f_{\text {hom }}^{q}$ follow from Proposition 3.1 and Proposition 7.1.

The proof of the theorem follows the same outlines of the one of Theorem 6.2. We sketch it by emphasizing the main differences.

Let $\Omega, \lambda, r, \beta, z_{0}, c$ be as above. Then, by using Theorem 5.2 in place of Theorem 4.4 , we first prove that

$$
\begin{aligned}
& \Gamma^{-}\left(L^{1}(\Omega)\right) \lim _{h \rightarrow+\infty}\left\{F_{0, h}\left(\Omega, \partial \Omega, u_{z_{0}}+c, u\right)+\lambda \int_{\Omega}|u|^{r} \mathrm{~d} x\right\} \\
& =\left\{\begin{array}{cl}
\int_{\Omega} \mathrm{sc}^{-} f_{\text {hom }}^{q}(\nabla u) \mathrm{d} x+\int_{\Omega}\left(\mathrm{sc}^{-} f_{\text {hom }}^{q}\right)^{\infty}\left(\frac{\mathrm{d} D^{s} u}{\mathrm{~d} \mid D^{s} u}\right) \mathrm{d}\left|D^{s} u\right| & \\
+\infty \quad+\int_{\partial \Omega}\left(\mathrm{sc}^{-} f_{\text {hom }}^{q}\right)^{\infty}\left(\left(u_{z_{0}}+c-u\right) \mathbf{n}_{\Omega}\right) \mathrm{d} \mathcal{H}^{n-1}+\lambda \int_{\Omega}|u|^{r} \mathrm{~d} x & \text { if } u \in B V(\Omega) \\
\text { for every } u \in L_{\text {loc }}^{1}\left(\mathbf{R}^{n}\right), & \text { if } u \in L_{\text {loc }}^{1}\left(\mathbf{R}^{n}\right) \backslash B V(\Omega)
\end{array}\right.
\end{aligned}
$$

where, for every $h \in \mathbf{N}, F_{0, h}\left(\Omega, \partial \Omega, u_{z_{0}}+c, \cdot\right)$ is defined by (2.21).

We now observe that (7.1) with $p=1$ ensures that the functionals $u \in L_{\text {loc }}^{1}\left(\mathbf{R}^{n}\right) \mapsto F_{0, h}(\Omega, \partial \Omega$, $\left.u_{z_{0}}+c, u\right)+\lambda \int_{\Omega}|u|^{r} \mathrm{~d} x+\int_{\Omega} \beta u \mathrm{~d} x$ are equicoercive once $L_{\text {loc }}^{1}\left(\mathbf{R}^{n}\right)$ is equipped with the $L^{1}(\Omega)$-topology.

Therefore the theorem follows by (7.14), Theorem 2.7, and by the $L^{1}(\Omega)$-continuity of the functional $u \in$ $L_{\mathrm{loc}}^{1}\left(\mathbf{R}^{n}\right) \mapsto \int_{\Omega} \beta u \mathrm{~d} x$

The following result deals with the case of Dirichlet minimum problems in Sobolev spaces.

Theorem 7.5. Let $f$ be as in (1.1) and satisfy (7.1), let $p \in] 1,+\infty], q \in[p,+\infty]$, and let $f_{\text {hom }}^{q}$ be defined in (1.5). Let $C \subseteq \mathbf{R}^{n}$ be convex such that (1.2) $\div(1.4)$ hold. For every $h \in \mathbf{N}, \Omega \in \mathcal{A}_{0}$ convex, $\beta \in L^{p^{\prime}}(\Omega)$, $z_{0} \in \operatorname{int}(C), c \in \mathbf{R}$ let

$$
\begin{gathered}
i_{h}^{D}(q, \Omega, \beta)=\inf \left\{\int_{\Omega} f(h x, \nabla u) \mathrm{d} x+\int_{\Omega} \beta u \mathrm{~d} x: u \in u_{z_{0}}+c+W_{0}^{1, q}(\Omega)\right\}, \\
m_{\infty}^{D}(q, \Omega, \beta)=\min \left\{\int_{\Omega} \operatorname{sc}^{-} f_{\mathrm{hom}}^{q}(\nabla u) \mathrm{d} x+\int_{\Omega} \beta u \mathrm{~d} x: u \in u_{z_{0}}+c+W_{0}^{1, p}(\Omega)\right\},
\end{gathered}
$$


and let $\left\{\tilde{u}_{h}\right\} \subseteq u_{z_{0}}+c+W_{0}^{1, q}(\Omega)$ be such that

$$
\lim _{h \rightarrow+\infty}\left(\int_{\Omega} f\left(h x, \nabla \tilde{u}_{h}\right) \mathrm{d} x+\int_{\Omega} \beta \tilde{u}_{h} \mathrm{~d} x-i_{h}^{D}(q, \Omega, \beta)\right)=0 .
$$

Then $f_{\mathrm{hom}}^{q}$ is convex and satisfies (6.2), $\left\{i_{h}^{D}(q, \Omega, \beta)\right\}$ converges to $m_{\infty}^{D}(q, \Omega, \beta),\left\{\tilde{u}_{h}\right\}$ is compact in $L^{p}(\Omega)$, and its converging subsequences converge to solutions of $m_{\infty}^{D}(q, \Omega, \beta)$.

Moreover, if $q=p$ and (1.9) too holds, then $\mathrm{sc}^{-} f_{\mathrm{hom}}^{p}=f_{\mathrm{hom}}^{p}$, for every $z \in \mathbf{R}^{n}$ the infimum in the definition of $f_{\text {hom }}^{p}(z)$ is attained, problems in (7.15) have solutions, and for every $h \in \mathbf{N}$ one can take $\tilde{u}_{h}$ as a minimizer of $i_{h}^{D}(q, \Omega, \beta)$.

Proof. The properties of $f_{\text {hom }}^{q}$ follow from Proposition 3.1, Proposition 7.1, and Proposition 3.4.

The proof of the theorem follows the same outlines of the one of Theorem 7.3. We sketch it by emphasizing the main differences.

Let $\Omega, \beta, z_{0}, c$ be as above, and let $P(\Omega, \cdot)$ is defined by

$$
P(\Omega, \cdot): u \in L_{\mathrm{loc}}^{1}\left(\mathbf{R}^{n}\right) \mapsto \begin{cases}\int_{\Omega} \beta u \mathrm{~d} x & \text { if } u \in W^{1, p}(\Omega) \\ +\infty & \text { if } u \in L_{\mathrm{loc}}^{1}\left(\mathbf{R}^{n}\right) \backslash W^{1, p}(\Omega) .\end{cases}
$$

Then, by using Theorem 5.2 in place of Theorem 4.4, we first prove that

$$
\begin{gathered}
\Gamma^{-}\left(L^{1}(\Omega)\right) \lim _{h \rightarrow+\infty}\left\{F_{0, h}\left(\Omega, \partial \Omega, u_{z_{0}}+c, u\right)+P(\Omega, u)\right\} \\
=\left\{\begin{array}{ll}
\int_{\Omega} \mathrm{sc}^{-} f_{\mathrm{hom}}^{q}(\nabla u) \mathrm{d} x+\int_{\Omega} \beta u \mathrm{~d} x & \text { if } u \in u_{z_{0}}+c+W^{1, p}(\Omega) \\
+\infty & \text { if } u \in L_{\mathrm{loc}}^{1}\left(\mathbf{R}^{n}\right) \backslash\left(u_{z_{0}}+c+W^{1, p}(\Omega)\right)
\end{array} \text { for every } u \in L_{\mathrm{loc}}^{1}\left(\mathbf{R}^{n}\right),\right.
\end{gathered}
$$

where, for every $h \in \mathbf{N}, F_{0, h}\left(\Omega, \partial \Omega, u_{z_{0}}+c, \cdot\right)$ is defined by $(2.21)$.

At this point, the same inequalities (with $\lambda=0$ ) as those used in the proof of Theorem 7.3, together with Poincaré inequality, ensure that the functionals $u \in L_{\mathrm{loc}}^{1}\left(\mathbf{R}^{n}\right) \mapsto F_{0, h}\left(\Omega, \partial \Omega, u_{z_{0}}+c, u\right)+P(\Omega, u)$ are equicoercive once $L_{\text {loc }}^{1}\left(\mathbf{R}^{n}\right)$ is equipped with the $L^{1}(\Omega)$-topology.

The proof now completes as in the one of Theorem 7.3, by using (7.16) in place of (7.8).

Eventually, we treat the case of mixed minimum problems.

Theorem 7.6. Let $f$ be as in (1.1), and let $f_{\mathrm{hom}}^{1}$ be defined in (1.5) with $q=1$. Assume that (1.10) holds with $\phi: \mathbf{R}^{n} \rightarrow[0,+\infty]$ convex and satisfying $\operatorname{int}(\operatorname{dom} \phi) \neq \emptyset, \lim _{z \rightarrow \infty} \frac{\phi(z)}{|z|}=+\infty, a \in L_{\mathrm{loc}}^{1}\left(\mathbf{R}^{n}\right) Y$-periodic, and $M \geq 0$. For every $h \in \mathbf{N}, \Omega \in \mathcal{A}_{0}$ convex, $\Gamma \subseteq \partial \Omega$ with $\mathcal{H}^{n-1}(\Gamma)>0, \beta \in L^{\infty}(\Omega), \gamma \in L^{\infty}(\partial \Omega)$, $z_{0} \in \operatorname{int}(\operatorname{dom} \phi), c \in \mathbf{R}$ let

$$
\begin{aligned}
& i_{h}^{M}(\Omega, \Gamma, \beta, \gamma)=\inf \left\{\int_{\Omega} f(h x, \nabla u) \mathrm{d} x+\int_{\Omega} \beta u \mathrm{~d} x+\int_{\partial \Omega} \gamma u \mathrm{~d} \mathcal{H}^{n-1}: u \in u_{z_{0}}+c+W_{0, \Gamma}^{1,1}(\Omega)\right\}, \\
& m_{\infty}^{M}(\Omega, \Gamma, \beta, \gamma)=\min \left\{\int_{\Omega} \operatorname{sc}^{-} f_{\mathrm{hom}}^{1}(\nabla u) \mathrm{d} x+\int_{\Omega} \beta u \mathrm{~d} x+\int_{\partial \Omega} \gamma u \mathrm{~d} \mathcal{H}^{n-1}: u \in u_{z_{0}}+c+W_{0, \Gamma}^{1,1}(\Omega)\right\},
\end{aligned}
$$

and let $\left\{\tilde{u}_{h}\right\} \subseteq u_{z_{0}}+c+W_{0, \Gamma}^{1,1}(\Omega)$ be such that

$$
\lim _{h \rightarrow+\infty}\left(\int_{\Omega} f\left(h x, \nabla \tilde{u}_{h}\right) \mathrm{d} x+\int_{\Omega} \beta \tilde{u}_{h} \mathrm{~d} x+\int_{\partial \Omega} \gamma \tilde{u}_{h} \mathrm{~d} \mathcal{H}^{n-1}-i_{h}^{M}(\Omega, \Gamma, \beta, \gamma)\right)=0 .
$$

Then $f_{\mathrm{hom}}^{1}$ is convex and satisfies

$$
\operatorname{sc}^{-} \phi(z) \leq \operatorname{sc}^{-} f_{\text {hom }}^{1}(z) \leq \int_{Y} a(y) \mathrm{d} y+M \mathrm{sc}^{-} \phi(z) \text { for every } z \in \mathbf{R}^{n},
$$


$\left\{i_{h}^{M}(\Omega, \Gamma, \beta, \gamma)\right\}$ converges to $m_{\infty}^{M}(\Omega, \Gamma, \beta, \gamma),\left\{\tilde{u}_{h}\right\}$ is compact in $L^{1}(\Omega)$, and its converging subsequences converge to solutions of $m_{\infty}^{M}(\Omega, \Gamma, \beta, \gamma)$.

Moreover, if (1.9) too holds, then $\mathrm{sc}^{-} f_{\mathrm{hom}}^{1}=f_{\mathrm{hom}}^{1}$, for every $z \in \mathbf{R}^{n}$ the infimum in the definition of $f_{\mathrm{hom}}^{1}(z)$ is attained, problems in (7.17) have solutions, and for every $h \in \mathbf{N}$ one can take $\tilde{u}_{h}$ as a solution of $i_{h}^{M}(\Omega, \Gamma, \beta, \gamma)$.

Proof. The properties of $f_{\text {hom }}^{1}$ and (7.18) follow from Proposition 3.1, Proposition 7.1, Proposition 3.4, and Proposition 3.3.

Let $\Omega, \Gamma, \beta, \gamma, z_{0}, c$ be as above, and let $P(\Omega, \cdot)$ be defined by

$$
P(\Omega, \cdot): u \in L_{\mathrm{loc}}^{1}\left(\mathbf{R}^{n}\right) \mapsto \begin{cases}\int_{\Omega} \beta u \mathrm{~d} x+\int_{\partial \Omega} \gamma u \mathrm{~d} \mathcal{H}^{n-1} & \text { if } u \in W^{1,1}(\Omega) \\ +\infty & \text { if } u \in L_{\mathrm{loc}}^{1}\left(\mathbf{R}^{n}\right) \backslash W^{1,1}(\Omega) .\end{cases}
$$

Then,

$$
\begin{gathered}
\Gamma^{-}\left(L^{1}(\Omega)\right) \lim _{h \rightarrow+\infty}\left\{F_{0, h}\left(\Omega, \Gamma, u_{z_{0}}+c, u\right)+P(\Omega, u)\right\} \\
= \begin{cases}\int_{\Omega} \operatorname{sc}^{-} f_{\text {hom }}^{q}(\nabla u) \mathrm{d} x+\int_{\Omega} \beta u \mathrm{~d} x+\int_{\partial \Omega} \gamma u \mathrm{~d} \mathcal{H}^{n-1} & \text { if } u \in u_{z_{0}}+c+W_{0, \Gamma}^{1,1}(\Omega) \\
+\infty & \text { if } u \in L_{\mathrm{loc}}^{1}\left(\mathbf{R}^{n}\right) \backslash\left(u_{z_{0}}+c+W_{0, \Gamma}^{1,1}(\Omega)\right)\end{cases}
\end{gathered}
$$

$$
\text { for every } u \in L_{\text {loc }}^{1}\left(\mathbf{R}^{n}\right) \text {, }
$$

where, for every $h \in \mathbf{N}, F_{0, h}\left(\Omega, \Gamma, u_{z_{0}}+c, \cdot\right)$ is defined by $(2.21)$.

To do this, we take $u \in u_{z_{0}}+c+W_{0, \Gamma}^{1,1}(\Omega)$ such that $\int_{\Omega} \operatorname{sc}^{-} f_{\text {hom }}^{q}(\nabla u) \mathrm{d} x+\int_{\Omega} \beta u \mathrm{~d} x+\int_{\partial \Omega} \gamma u \mathrm{~d} \mathcal{H}^{n-1}$ $<+\infty$. Then, Theorem 6.1 provides $\left\{u_{h}\right\} \subseteq u_{z_{0}}+c+W_{0, \Gamma}^{1,1}(\Omega)$ such that $u_{h} \rightarrow u$ in $L^{1}(\Omega)$, and

$$
\int_{\Omega} \mathrm{sc}^{-} f_{\text {hom }}^{q}(\nabla u) \mathrm{d} x=\limsup _{h \rightarrow+\infty} \int_{\Omega} f\left(h x, \nabla u_{h}\right) \mathrm{d} x
$$

Now, by (1.10) and the de la Vallée Poussin Compactness Theorem, it turns out that $u_{h} \rightarrow u$ in weak- $W^{1,1}(\Omega)$. Therefore, the continuity of $v \in W^{1,1}(\Omega) \mapsto \int_{\Omega} \beta v \mathrm{~d} x+\int_{\partial \Omega} \gamma v \mathrm{~d} \mathcal{H}^{n-1}$ with respect to the weak- $W^{1,1}(\Omega)$-topology yields that

$$
\begin{gathered}
\Gamma^{-}\left(L^{1}(\Omega)\right) \limsup _{h \rightarrow+\infty}\left\{F_{0, h}\left(\Omega, \Gamma, u_{z_{0}}+c, u\right)+P(\Omega, u)\right\} \\
\leq \limsup _{h \rightarrow+\infty}\left\{\int_{\Omega} f\left(h x, \nabla u_{h}\right) \mathrm{d} x+\int_{\Omega} \beta u_{h} \mathrm{~d} x+\int_{\partial \Omega} \gamma u_{h} \mathrm{~d} \mathcal{H}^{n-1}\right\}=\int_{\Omega} \mathrm{sc}^{-} f_{\text {hom }}^{q}(\nabla u) \mathrm{d} x+\int_{\Omega} \beta u \mathrm{~d} x+\int_{\partial \Omega} \gamma u \mathrm{~d} \mathcal{H}^{n-1} \\
\text { for every } u \in W^{1, p}(\Omega) .
\end{gathered}
$$

On the other side, if $u \in L_{\text {loc }}^{1}\left(\mathbf{R}^{n}\right)$ satisfies $\Gamma^{-}\left(L^{1}(\Omega)\right) \liminf _{h \rightarrow+\infty}\left\{F_{0, h}\left(\Omega, \Gamma, u_{z_{0}}+c, u\right)+P(\Omega, u)\right\}<+\infty$, there exists $\left\{u_{h}\right\} \subseteq L_{\text {loc }}^{1}\left(\mathbf{R}^{n}\right)$ and $\left\{h_{k}\right\} \subseteq \mathbf{N}$ strictly increasing, such that $u_{h} \rightarrow u$ in $L^{1}(\Omega),\left\{u_{h_{k}}\right\} \subseteq u_{z_{0}}+c+$ $W_{0, \Gamma}^{1,1}(\Omega)$, and

$$
\begin{gathered}
\Gamma^{-}\left(L^{1}(\Omega)\right) \liminf _{h \rightarrow+\infty}\left\{F_{0, h}\left(\Omega, \Gamma, u_{z_{0}}+c, u\right)+P(\Omega, u)\right\} \\
=\liminf _{k \rightarrow+\infty}\left\{\int_{\Omega} f\left(h_{k} x, \nabla u_{h_{k}}\right) \mathrm{d} x+\int_{\Omega} \beta u_{h_{k}} \mathrm{~d} x+\int_{\partial \Omega} \gamma u_{h_{k}} \mathrm{~d} \mathcal{H}^{n-1}\right\} .
\end{gathered}
$$

Then, the same coerciveness arguments exploited above provide that $u \in W^{1,1}(\Omega)$ and that $u_{h_{k}} \rightarrow u$ in weak$W^{1,1}(\Omega)$. Moreover the weak- $W^{1,1}(\Omega)$-closedness of $W_{0, \Gamma}^{1,1}(\Omega)$ also implies that actually $u \in u_{z_{0}}+c+W_{0, \Gamma}^{1,1}(\Omega)$. Consequently, if for every $h \in \mathbf{N} F_{h}(\Omega, \cdot)$ is defined by (2.12), then (7.21), the above continuity arguments, and Theorem 4.4 imply that

$$
\Gamma^{-}\left(L^{1}(\Omega)\right) \liminf _{h \rightarrow+\infty}\left\{F_{0, h}\left(\Omega, \Gamma, u_{z_{0}}+c, u\right)+P(\Omega, u)\right\}
$$




$$
\begin{aligned}
& \geq \liminf _{k \rightarrow+\infty} \int_{\Omega} f\left(h_{k} x, \nabla u_{h_{k}}\right) \mathrm{d} x+\int_{\Omega} \beta u \mathrm{~d} x+\int_{\partial \Omega} \gamma u \mathrm{~d} \mathcal{H}^{n-1} \geq \liminf _{h \rightarrow+\infty} F_{h}\left(\Omega, u_{h}\right)+\int_{\Omega} \beta u \mathrm{~d} x+\int_{\partial \Omega} \gamma u \mathrm{~d} \mathcal{H}^{n-1} \\
& \quad \geq \begin{cases}\int_{\Omega} \mathrm{sc}^{-} f_{\text {hom }}^{q}(\nabla u) \mathrm{d} x+\int_{\Omega} \beta u \mathrm{~d} x+\int_{\partial \Omega} \gamma u \mathrm{~d} \mathcal{H}^{n-1} & \text { if } u \in u_{z_{0}}+c+W_{0, \Gamma}^{1,1}(\Omega) \\
+\infty & \text { if } u \in L_{\text {loc }}^{1}\left(\mathbf{R}^{n}\right) \backslash\left(u_{z_{0}}+c+W_{0, \Gamma}^{1,1}(\Omega)\right),\end{cases}
\end{aligned}
$$

for every $u \in L_{\text {loc }}^{1}\left(\mathbf{R}^{n}\right)$.

By (7.20) and (7.22), equality (7.19) follows.

We now observe that (1.10) ensures that the functionals $u \in L_{\text {loc }}^{1}\left(\mathbf{R}^{n}\right) \mapsto F_{0, h}\left(\Omega, \Gamma, u_{z_{0}}+c, u\right)+P(\Omega, u)$ are equicoercive once $L_{\text {loc }}^{1}\left(\mathbf{R}^{n}\right)$ is equipped with the $L^{1}(\Omega)$-topology.

In fact, by (7.1), Hölder Inequality, and Sobolev Imbedding Theorem it is easy to see that there exists $\left.C_{\Omega} \in\right] 0,+\infty\left[\right.$ such that (we denote here by $C_{\Omega}$ a constant depending only on $\Omega$ )

$$
\begin{aligned}
& F_{0, h}\left(\Omega, \Gamma, u_{z_{0}}+c, u\right)+P(\Omega, u) \geq \int_{\Omega} \phi(\nabla u) \mathrm{d} x-\|\beta\|_{L^{\infty}(\Omega)}\|u\|_{L^{1}(\Omega)}-\|\gamma\|_{L^{\infty}(\partial \Omega)}\|u\|_{L^{1}(\partial \Omega)} \\
& \geq \int_{\Omega} \phi(\nabla u) \mathrm{d} x-C_{\Omega}\left(\|\beta\|_{L^{\infty}(\Omega)}+\|\gamma\|_{L^{\infty}(\partial \Omega)}\right)\|u\|_{W^{1,1}(\Omega)} \text { for every } h \in \mathbf{N}, u \in W_{\mathrm{loc}}^{1,1}(\Omega),
\end{aligned}
$$

from which, together with the superlinearity properties of $\phi,(2.20)$, and the compact embedding of $W^{1,1}(\Omega)$ in $L^{1}(\Omega)$, the desired equicoerciveness follows.

Therefore the theorem follows by (7.19) and Theorem 2.7 (applied with $E \equiv 0$ ).

Finally, if (1.9) holds, the existence of the solutions of the problems in (7.17) follows from the above coerciveness argument and Theorem 2.5.

Acknowledgements. This work has been produced with the financial support of the European Commission through the Human Potential Programme (Contract HPRN-CT-2000-00109). It has also been produced in the framework of the project "Modelli e metodi di ottimizzazione: aspetti teorici e computazionali" of the Italian CNR program "Agenzia2000", and of the project "UFI401KXX7-Programme Vinci 2001 de l'Université franco-italienne".

\section{REFERENCES}

[1] L. Ambrosio, N. Fusco and D. Pallara, Functions of Bounded Variation and Free Discontinuity Problems. Claredon Press, Oxford Math. Monogr. (2000).

[2] A.H.T. Banks, N.J. Lybeck, B. Munoz and L. Yanyo, Nonlinear Elastomers: Modeling and Estimation, in Proc. of the "Third IEEE Mediterranean Symposium on New Directions in Control and Automation", Vol. 1. Limassol, Cyprus (1995) 1-7.

[3] A. Bensoussan, J.L. Lions and G. Papanicolaou, Asymptotic Analysis for Periodic Structures. North Holland, Stud. Math. Appl. 5 (1978).

[4] A. Braides and A. Defranceschi, Homogenization of Multiple Integrals. Oxford University Press, Oxford Lecture Ser. Math. Appl. 12 (1998).

[5] G. Buttazzo, Semicontinuity, Relaxation and Integral Representation in the Calculus of Variations. Longman Scientific \& Technical, Pitman Res. Notes Math. Ser. 207 (1989).

[6] L. Carbone, D. Cioranescu, R. De Arcangelis and A. Gaudiello, An Approach to the Homogenization of Nonlinear Elastomers via the Theory of Unbounded Functionals. C. R. Acad. Sci. Paris Sér. I Math. 332 (2001) 283-288.

[7] L. Carbone, D. Cioranescu, R. De Arcangelis and A. Gaudiello, Homogenization of Unbounded Functionals and Nonlinear Elastomers. The General Case. Asymptot. Anal. 29 (2002) 221-272.

[8] L. Carbone, D. Cioranescu, R. De Arcangelis and A. Gaudiello, An Approach to the Homogenization of Nonlinear Elastomers in the Case of the Fixed Constraints Set. Rend. Accad. Sci. Fis. Mat. Napoli (4) 67 (2000) 235-244.

[9] L. Carbone and R. De Arcangelis, On Integral Representation, Relaxation and Homogenization for Unbounded Functionals. Atti Accad. Naz. Lincei Cl. Sci. Fis. Mat. Natur. Rend. Lincei (9) Mat. Appl. 8 (1997) 129-135.

[10] L. Carbone and R. De Arcangelis, On the Relaxation of Some Classes of Unbounded Integral Functionals. Matematiche 51 (1996) 221-256; Special Issue in honor of Francesco Guglielmino.

[11] L. Carbone and R. De Arcangelis, Unbounded Functionals: Applications to the Homogenization of Gradient Constrained Problems. Ricerche Mat. 48-Suppl. (1999) 139-182.

[12] L. Carbone and R. De Arcangelis, On the Relaxation of Dirichlet Minimum Problems for Some Classes of Unbounded Integral Functionals. Ricerche Mat. 48 (1999) 347-372; Special Issue in memory of Ennio De Giorgi. 
[13] L. Carbone and R. De Arcangelis, On the Unique Extension Problem for Functionals of the Calculus of Variations. Atti Accad. Naz. Lincei Cl. Sci. Fis. Mat. Natur. Rend. Lincei (9) Mat. Appl. 12 (2001) 85-106.

[14] L. Carbone and S. Salerno, Further Results on a Problem of Homogenization with Constraints on the Gradient. J. Analyse Math. 44 (1984/85) 1-20.

[15] D. Cioranescu and P. Donato, An Introduction to Homogenization. Oxford University Press, Oxford Lecture Ser. Math. Appl. 17 (1999).

[16] A. Corbo Esposito and R. De Arcangelis, The Lavrentieff Phenomenon and Different Processes of Homogenization. Comm. Partial Differential Equations 17 (1992) 1503-1538.

[17] A. Corbo Esposito and R. De Arcangelis, Homogenization of Dirichlet Problems with Nonnegative Bounded Constraints on the Gradient. J. Analyse Math. 64 (1994) 53-96.

[18] A. Corbo Esposito and F. Serra Cassano, A Lavrentieff Phenomenon for Problems of Homogenization with Constraints on the Gradient. Ricerche Mat. 46 (1997) 127-159.

[19] G. Dal Maso, An Introduction to Г-Convergence. Birkhäuser-Verlag, Progr. Nonlinear Differential Equations Appl. 8 (1993).

[20] C. D'Apice, T. Durante and A. Gaudiello, Some New Results on a Lavrentieff Phenomenon for Problems of Homogenization with Constraints on the Gradient. Matematiche 54 (1999) 3-47.

[21] E. De Giorgi and T. Franzoni, Su un tipo di convergenza variazionale. Atti Accad. Naz. Lincei Rend. Cl. Sci. Fis. Mat. Natur. (8) 58 (1975) 842-850.

[22] G. Duvaut and J.L. Lions, Inequalities in Mechanics and Physics. Springer-Verlag, Grundlehren Math. Wiss. 219 (1976).

[23] R.T. Rockafellar, Convex Analysis. Princeton University Press, Princeton Math. Ser. 28 (1972).

[24] L.R.G. Treloar, The Physics of Rubber Elasticity. Clarendon Press, Oxford, First Ed. (1949), Third Ed. (1975).

[25] W.P. Ziemer, Weakly Differentiable Functions. Springer-Verlag, Grad. Texts in Math. 120 (1989). 